\title{
Review Article \\ Existence for Singular Periodic Problems: A Survey of Recent Results
}

\author{
Jifeng Chu, ${ }^{1}$ Juntao Sun, ${ }^{2}$ and Patricia J. Y. Wong ${ }^{3}$ \\ ${ }^{1}$ Department of Mathematics, College of Science, Hohai University, Nanjing 210098, China \\ ${ }^{2}$ School of Science, Shandong University of Technology, Zibo, Shandong 255049, China \\ ${ }^{3}$ School of Electrical and Electronic Engineering, Nanyang Technological University, 50 Nanyang Avenue, Singapore 639798 \\ Correspondence should be addressed to Jifeng Chu; jifengchu@126.com
}

Received 13 December 2012; Accepted 11 January 2013

Academic Editor: Yonghui Xia

Copyright (C) 2013 Jifeng Chu et al. This is an open access article distributed under the Creative Commons Attribution License, which permits unrestricted use, distribution, and reproduction in any medium, provided the original work is properly cited.

We present a survey on the existence of periodic solutions of singular differential equations. In particular, we pay our attention to singular scalar differential equations, singular damped differential equations, singular impulsive differential equations, and singular differential systems.

\section{Introduction}

During the last two decades, singular differential equations have attracted many researchers [1-11] because such equations describe many problems in the applied sciences, such as the Brillouin focusing system [12-14], nonlinear elasticity [15], and gravitational forces [3]. Besides these important applications, it has been found that a particular case of singular equations, the Ermakov-Pinney equation, plays an important role in studying the Lyapunov stability of periodic solutions of Lagrangian equations [16-18].

In the literature, two different approaches have been used to establish the existence results for singular equations. The first one is the variational approach $[3,4,6,19,20]$ and the second one is topological methods [1, 10, 21-28]. In our opinion, the first important result was proved in the pioneering paper of Lazer and Solimini [29]. They proved that a necessary and sufficient condition for the existence of a positive periodic solution for

$$
x^{\prime \prime}=\frac{1}{x^{\lambda}}+e(t)
$$

is that the mean value of $e$ is negative; that is, $\bar{e}<0$, here $\lambda \geq 1$, which corresponds to a strong force condition, according to a terminology first introduced by Gordon [30]. Moreover, if $0<\lambda<1$, which corresponds to a weak force condition, they found examples of functions $e$ with negative mean values and yet no periodic solutions exist. Therefore, there is an essential difference between a strong singularity and a weak singularity. Since the work of Lazer and Solimini, the strong force condition became standard in related work, see, for instance, $[8,15,18,27,28]$. Compared with the case of a strong singularity, the study of the existence of periodic solutions under the presence of a weak singularity is more recent, but it has also attracted many researchers [31-39]. In [39], for the first time in this topic, Torres et al. proved an existence result which is valid for a weak singularity, whereas the validity of such results under a strong force assumption remains as an open problem, which was partially solved in [32].

The main aim of this survey is to present some recent existence results for singular differential equations. In particular, we will consider the scalar singular equations, singular damped equations, singular impulsive equations, and singular differential systems. We will also include some examples to illustrate the results presented.

The rest of this paper is organized as follows. In Section 2, we will state some important results for the second-order scalar singular differential equations. Singular damped equations will be considered in Section 3. In Section 4, singular impulsive differential equations will be studied. Finally in Section 5, we will focus on the singular differential systems. Sections 2 and 3 are mainly written by the first author. Section 4 is mainly written by the second author, and Section 5 is mainly completed by the third author. 
All the results presented in Sections 3-5 shed some lights on the differences between a strong singularity and a weak singularity.

Finally in this section, we must note that besides the results presented in this survey, many interesting and important results on singular differential equations have been obtained by other researchers, see, for example, [9, 40-45] and the references cited therein.

In this paper, we denote the essential supremum and infimum of $p$ by $p^{*}$ and $p_{*}$, respectively, for a given function $p \in L^{1}[0, T]$ essentially bounded.

\section{Second-Order Scalar Singular Equations}

In this section, we recall some results for second-order singular differential equations

$$
x^{\prime \prime}+a(t) x=f(t, x)+e(t),
$$

here $a(t), e(t)$ are continuous, $T$-periodic functions. The nonlinearity $f(t, x)$ is continuous in $(t, x)$ and $T$-periodic in $t$ and has a singularity at $x=0$.

First we need to present some preliminary results on the linear equation

$$
x^{\prime \prime}+a(t) x=p(t)
$$

with periodic boundary conditions

$$
x(0)=x(T), \quad x^{\prime}(0)=x^{\prime}(T) .
$$

We assume the following:

(A) the Green function $G(t, s)$, associated with (3)-(4), is positive for all $(t, s) \in[0, T] \times[0, T]$, or

(B) the Green function $G(t, s)$, associated with (3)-(4), is nonnegative for all $(t, s) \in[0, T] \times[0, T]$.

When $a(t)=k^{2}$, condition (A) is equivalent to $0<k^{2}<$ $\lambda_{1}=(\pi / T)^{2}$ and condition (B) is equivalent to $0<k^{2} \leq \lambda_{1}$. In this case, we have

$$
G(t, s)= \begin{cases}\frac{\sin k(t-s)+\sin k(T-t+s)}{2 k(1-\cos k T)}, & 0 \leq s \leq t \leq T, \\ \frac{\sin k(s-t)+\sin k(T-s+t)}{2 k(1-\cos k T)}, & 0 \leq t \leq s \leq T .\end{cases}
$$

For a nonconstant function $a(t)$, there is an $L^{p}$-criterion proved in [46], which is given in Lemma 1 for the sake of completeness. Let $\mathbf{K}(q)$ denote the best Sobolev constant in the following inequality:

$$
C\|u\|_{q}^{2} \leq\left\|u^{\prime}\right\|_{2}^{2}, \quad \forall u \in H_{0}^{1}(0, T) .
$$

The explicit formula for $\mathbf{K}(q)$ is

$$
\mathbf{K}(q)=\left\{\begin{array}{cc}
\frac{2 \pi}{q T^{1+2 / q}}\left(\frac{2}{2+q}\right)^{1-2 / q}\left(\frac{\Gamma(1 / q)}{\Gamma(1 / 2+1 / q)}\right)^{2} \\
\text { if } 1 \leq q<\infty, \\
\frac{4}{T} & \text { if } q=\infty,
\end{array}\right.
$$

where $\Gamma$ is the gamma function, see $[47,48]$.
Lemma 1 (see [46, Corollary 2.3]). Assume that $a(t)>0$ and $a \in L^{p}[0, T]$ for some $1 \leq p \leq \infty$. If

$$
\|a\|_{p}<\mathbf{K}(2 \widetilde{p})
$$

then the condition (A) holds. Moreover, condition (B) holds if

$$
\|a\|_{p} \leq \mathbf{K}(2 \widetilde{p})
$$

When the hypothesis $(\mathrm{A})$ is satisfied, we denote

$$
m=\min _{0 \leq s, t \leq T} G(t, s), \quad M=\max _{0 \leq s, t \leq T} G(t, s), \quad \sigma=\frac{m}{M} .
$$

Obviously, $M>m>0$ and $0<\sigma<1$.

The first existence result deals with the case of a strong singularity and the proof is based on the following nonlinear alternative of Leray-Schauder, which can be found in [49] or [50, pages $120-130]$.

Lemma 2. Assume $\Omega$ is an open subset of a convex set $K$ in a normed linear space $X$ and $p \in \Omega$. Let $T: \bar{\Omega} \rightarrow K$ be a compact and continuous map. Then one of the following two conclusions holds.

(I) Thas at least one fixed point in $\bar{\Omega}$.

(II) There exists $x \in \partial \Omega$ and $0<\lambda<1$ such that $x=$ $\lambda T x+(1-\lambda) p$.

Theorem 3 (see [37, Theorem 4.1]). Suppose that a( $t$ ) satisfies (A) and $f(t, x)$ satisfies the following.

$\left(\mathrm{H}_{1}\right)$ There exists a nonincreasing positive continuous function $g_{0}(x)$ on $(0, \infty)$ and a constant $R_{0}>0$ such that $f(t, x) \geq g_{0}(x)$ for $(t, x) \in[0, T] \times\left(0, R_{0}\right]$, where $g_{0}(x)$ satisfies

$$
\lim _{x \rightarrow 0^{+}} g_{0}(x)=+\infty, \quad \lim _{x \rightarrow 0^{+}} \int_{x}^{R_{0}} g_{0}(u) d u=+\infty
$$

$\left(\mathrm{H}_{2}\right)$ There exist continuous, nonnegative functions $g(x)$ and $h(x)$ such that

$$
0 \leq f(t, x) \leq g(x)+h(x) \quad \forall(t, x) \in[0, T] \times(0, \infty),
$$

$g(x)>0$ is nonincreasing and $h(x) / g(x)$ is nondecreasing in $x \in(0, \infty)$.

$\left(\mathrm{H}_{3}\right)$ There exists a positive number $r$ such that $\sigma r+\gamma_{*}>0$ and

$$
\begin{aligned}
& \frac{r}{g\left(\sigma r+\gamma_{*}\right)\left\{1+\left(h\left(r+\gamma^{*}\right) / g\left(r+\gamma^{*}\right)\right)\right\}}>\omega^{*}, \\
& \text { here } \\
& \gamma(t)=\int_{0}^{T} G(t, s) e(s) d s, \quad \omega(t)=\int_{0}^{T} G(t, s) d s .
\end{aligned}
$$

Then for each $e \in \mathbb{C}(\mathbb{R} / T \mathbb{Z}, \mathbb{R})$, (2) has at least one positive periodic solution $x$ with $x(t)>\gamma(t)$ for all t and $0<\|x-\gamma\|<r$. 
Note that the study in [37, Theorem 4.1] is slightly different from the above presentation. However, the proof of the above theorem follows from that of [37, Theorem 4.1] with some minor necessary changes. Condition $\left(\mathrm{H}_{1}\right)$ corresponds to the classical strong force condition, which was first introduced by Gordon in [30]. In fact, condition $\left(\mathrm{H}_{1}\right)$ is only used when we try to obtain a prior lower bound. In Theorem 4, we will show that, for the case $\gamma_{*} \geq 0$, we can remove the strong force condition $\left(\mathrm{H}_{1}\right)$ and replace it by one weak force condition.

Theorem 4 (see [33, Theorem 3.1]). Assume that (A) and $\left(\mathrm{H}_{2}\right)-\left(\mathrm{H}_{3}\right)$ are satisfied. Suppose further the following condition.

$\left(\mathrm{H}_{4}\right)$ For each constant $L>0$, there exists a continuous function $\phi_{L}>0$ such that $f(t, x) \geq \phi_{L}(t)$ for all $(t, x) \in[0, T] \times(0, L]$.

Then for each $e(t)$ with $\gamma_{*} \geq 0$, (2) has at least one positive periodic solution $x$ with $x(t)>\gamma(t)$ for all t and $0<\|x-\gamma\|<r$.

For the superlinear case, we can establish the multiplicity result. The proof is based on a well-known fixed point theorem in cones, which can be found in [51]. Let $K$ be a cone in $X$ and $D$ is a subset of $X$, we write $D_{K}=D \cap K$ and $\partial_{K} D=(\partial D) \cap K$.

Theorem 5 (see [51]). Let $X$ be a Banach space and $K$ a cone in $X$. Assume $\Omega^{1}, \Omega^{2}$ are open bounded subsets of $X$ with $\Omega_{K}^{1} \neq \emptyset, \bar{\Omega}_{K}^{1} \subset \Omega_{K}^{2}$. Let

$$
T: \bar{\Omega}_{K}^{2} \longrightarrow K
$$

be a completely continuous operator such that

(a) $\|T x\| \leq\|x\|$ for $x \in \partial_{K} \Omega^{1}$,

(b) there exists $v \in K \backslash\{0\}$ such that $x \neq T x+$ $\lambda v$ forall $x \in \partial_{K} \Omega^{2}$ and all $\lambda>0$.

Then $T$ has a fixed point in $\bar{\Omega}_{K}^{2} \backslash \Omega_{K}^{1}$.

Theorem 6 (see [33, Theorem 3.2]). Suppose that a $(t)$ satisfies (A) and $f(t, x)$ satisfies $\left(\mathrm{H}_{2}\right)-\left(\mathrm{H}_{3}\right)$. Furthermore, assume the following conditions.

$\left(\mathrm{H}_{5}\right)$ There exist continuous, nonnegative functions $g_{1}(x), h_{1}(x)$ such that

$f(t, x) \geq g_{1}(x)+h_{1}(x), \quad \forall(t, x) \in[0, T] \times(0, \infty)$,

$g_{1}(x)>0$ is nonincreasing and $h_{1}(x) / g_{1}(x)$ is nondecreasing in $x$.

$\left(\mathrm{H}_{6}\right)$ There exists $R>0$ with $\sigma R>r$ such that

$$
\frac{\sigma R}{g_{1}\left(R+\gamma^{*}\right)\left\{1+\left(h_{1}\left(\sigma R+\gamma_{*}\right) / g_{1}\left(\sigma R+\gamma_{*}\right)\right)\right\}} \leq \omega_{*} .
$$

Then (2) has one positive periodic solution $\tilde{x}$ with $r<\|\widetilde{x}-\gamma\| \leq$ $R$.
Combined Theorems 3 and 4 with Theorem 6 , we can get the following two multiplicity results.

Theorem 7. Suppose that $a(t)$ satisfies $(A)$ and $f(t, x)$ satisfies $\left(H_{1}\right)-\left(H_{3}\right)$ and $\left(H_{5}\right)-\left(H_{6}\right)$. Then (2) has two different positive periodic solutions $x$ and $\tilde{x}$ with $0<\|x-\gamma\|<r<\|\widetilde{x}-\gamma\| \leq R$.

Theorem 8. Suppose that a $(t)$ satisfies $(A)$ and $f(t, x)$ satisfies $\left(H_{2}\right)-\left(H_{6}\right)$. Then (2) has two different positive periodic solutions $x$ and $\tilde{x}$ with $0<\|x-\gamma\|<r<\|\widetilde{x}-\gamma\| \leq R$.

To illustrate our results, we have selected the following singular equation:

$$
x^{\prime \prime}+a(t) x=x^{-\alpha}+\mu x^{\beta}+e(t),
$$

here $a, e \in \mathbb{C}[0, T], \alpha, \beta>0$, and $\mu \in \mathbb{R}$ is a given parameter. The corresponding results are also valid for the general case

$$
x^{\prime \prime}+a(t) x=\frac{b(t)}{x^{\alpha}}+\mu c(t) x^{\beta}+e(t),
$$

with $b, c \in \mathbb{C}[0, T]$.

Corollary 9. Assume that $a(t)$ satisfies (A) and $\alpha>0, \beta \geq$ $0, \mu>0$. Then one has the following results.

(i) If $\alpha \geq 1, \beta<1$, then for each $e \in \mathbb{C}(\mathbb{R} / T \mathbb{Z}, \mathbb{R})$, (18) has at least one positive periodic solution for all $\mu>0$.

(ii) If $\alpha \geq 1, \beta \geq 1$, then for each e $\in \mathbb{C}(\mathbb{R} / T \mathbb{Z}, \mathbb{R})$, (18) has at least one positive periodic solution for each $0<$ $\mu<\mu_{1}$; here $\mu_{1}$ is some positive constant.

(iii) If $\alpha \geq 1, \beta>1$, then for each $e \in \mathbb{C}(\mathbb{R} / T \mathbb{Z}$, $\mathbb{R})$, (18) has at least two positive periodic solutions for each $0<$ $\mu<\mu_{1}$.

(iv) If $\alpha>0, \beta<1$, then for each $e \in \mathbb{C}(\mathbb{R} / T \mathbb{Z}, \mathbb{R})$, with $\gamma_{*} \geq 0$, (18) has at least one positive periodic solution for all $\mu>0$.

(v) If $\alpha>0, \beta \geq 1$, then for each $e \in \mathbb{C}(\mathbb{R} / T \mathbb{Z}, \mathbb{R})$, with $\gamma_{*} \geq 0$, (18) has at least one positive periodic solution for each $0<\mu<\mu_{1}$.

(vi) If $\alpha>0, \beta>1$, then for each $e \in \mathbb{C}(\mathbb{R} / T \mathbb{Z}, \mathbb{R})$, with $\gamma_{*} \geq 0$, (18) has at least two positive periodic solutions for each $0<\mu<\mu_{1}$.

All the above results require that the linear equation satisfies (A), which cannot cover the critical case. The next few results deal with the case when the condition (B) is satisfied and the proof is based on Schauder's fixed point theorem. 
Theorem 10 (see [31, Theorem 3.1]). Assume that conditions (B) and $\left(\mathrm{H}_{2}\right)$ and $\left(\mathrm{H}_{4}\right)$ are satisfied. Furthermore, suppose that

$\left(\mathrm{H}_{7}\right)$ there exists a positive constant $R>0$ such that $R>$ $\Phi_{*}, \Phi_{*}+\gamma_{*}>0$ and

$$
R \geq g\left(\Phi_{*}+\gamma_{*}\right)\left\{1+\frac{h\left(R+\gamma^{*}\right)}{g\left(R+\gamma^{*}\right)}\right\} \omega^{*},
$$

here $\Phi_{*}=\min _{t} \Phi(t), \Phi(t)=\int_{0}^{T} G(t, s) \phi_{R+\gamma^{*}}(s) d s$.

Then (2) has at least one positive T-periodic solution.

As an application of Theorem 10, we consider the case $\gamma_{*}=0$. Corollary 11 is a direct result of Theorem 10 .

Corollary 11 (see [31, Corollary 3.2]). Assume that conditions (B) and $\left(\mathrm{H}_{2}\right)$ and $\left(\mathrm{H}_{4}\right)$ are satisfied. Furthermore, assume that

$\left(\mathrm{H}_{8}\right)$ there exists a positive constant $R>0$ such that $R>\Phi_{*}$ and

$$
R \geq g\left(\Phi_{*}\right)\left\{1+\frac{h\left(R+\gamma^{*}\right)}{g\left(R+\gamma^{*}\right)}\right\} \omega^{*}
$$

If $\gamma_{*}=0$, then (2) has at least one positive T-periodic solution.

Corollary 12 (see [31, Example 3.5]). Suppose that a satisfies (B) and $0<\alpha<1, \beta \geq 0$, then for each $e(t) \in \mathbb{C}(\mathbb{R} / T \mathbb{Z}, \mathbb{R})$, with $\gamma_{*}=0$, one has the following:

(i) if $\alpha+\beta<1-\alpha^{2}$, then (18) has at least one positive periodic solution for each $\mu \geq 0$,

(ii) if $\alpha+\beta \geq 1-\alpha^{2}$, then (18) has at least one positive T-periodic solution for each $0 \leq \mu<\mu_{2}$, where $\mu_{2}$ is some positive constant.

The next results explore the case when $\gamma_{*}>0$.

Theorem 13 (see [31, Theorem 3.6]). Suppose that a( $t$ ) satisfies (B) and $f(t, x)$ satisfies condition $\left(\mathrm{H}_{2}\right)$. Furthermore, assume that

$\left(\mathrm{H}_{9}\right)$ there exists $R>\gamma^{*}$ such that

$$
g\left(\gamma_{*}\right)\left\{1+\frac{h\left(R+\gamma^{*}\right)}{g\left(R+\gamma^{*}\right)}\right\} \omega^{*} \leq R .
$$

If $\gamma_{*}>0$, then (2) has at least one positive T-periodic solution.

Corollary 14 (see [31, Example 3.8]). Suppose that $a(t)$ satisfies $(B)$ and $\alpha, \beta \geq 0$, then for each $e \in \mathbb{C}(\mathbb{R} / T \mathbb{Z}, \mathbb{R})$, with $\gamma_{*}>0$, one has the following:

(i) if $\alpha+\beta<1$, then (18) has at least one positive $T$ periodic solution for each $\mu \geq 0$,

(ii) if $\alpha+\beta \geq 1$, then (18) has at least one positive $T$ periodic solution for each $0 \leq \mu<\mu_{3}$, where $\mu_{3}$ is some positive constant.

\section{Singular Damped Equations}

In this section, we recall some results on second-order singular damped differential equations

$$
x^{\prime \prime}+h(t) x^{\prime}+a(t) x=f\left(t, x, x^{\prime}\right),
$$

where $h, a \in \mathbb{C}(\mathbb{R} / T \mathbb{Z}, \mathbb{R})$ and the nonlinearity $f \in$ $\mathbb{C}((\mathbb{R} / T \mathbb{Z}) \times(0, \infty) \times \mathbb{R}, \mathbb{R})$. In particular, the nonlinearity may have a repulsive singularity at $x=0$, which means that

$$
\lim _{x \rightarrow 0^{+}} f(t, x, y)=+\infty, \quad \text { uniformly in }(t, y) \in \mathbb{R}^{2} \text {. }
$$

First we recall some results on the linear damped equation

$$
x^{\prime \prime}+h(t) x^{\prime}+a(t) x=0,
$$

associated to periodic boundary conditions (4). As in the last section, we say that (25)-(4) is nonresonant when its unique $T$-periodic solution is the trivial one. When (25)-(4) is nonresonant, as a consequence of Fredholm's alternative, the nonhomogeneous equation

$$
x^{\prime \prime}+h(t) x^{\prime}+a(t) x=l(t)
$$

admits a unique $T$-periodic solution which can be written as

$$
x(t)=\int_{0}^{T} G_{2}(t, s) l(s) d s,
$$

where $G_{2}(t, s)$ is the Green's function of problem (25)-(4). We also assume that the following standing hypothesis is satisfied.

(C) The Green's function $G_{2}(t, s)$, associated with (25)-(4), is positive for all $(t, s) \in[0, T] \times[0, T]$.

To guarantee that $(C)$ is satisfied, we require the antimaximum principle for (25)-(4) proved by Hakl and Torres in [52]. To do this, let us define the functions

$$
\begin{gathered}
\sigma(h)(t)=\exp \left(\int_{0}^{t} h(s) d s\right), \\
\sigma_{1}(h)(t)=\sigma(h)(T) \int_{0}^{t} \sigma(h)(s) d s+\int_{t}^{T} \sigma(h)(s) d s .
\end{gathered}
$$

Lemma 15 (see [52, Theorem 2.2]). Assume that $a \not \equiv 0$ and the following two inequalities are satisfied:

$$
\begin{gathered}
\int_{0}^{T} a(s) \sigma(h)(s) \sigma_{1}(-h)(s) d s \geq 0, \\
\sup _{0 \leq t \leq T}\left\{\int_{t}^{t+T} \sigma(-h)(s) d s \int_{t}^{t+T}[a(s)]+\sigma(h)(s) d s\right\} \leq 4,
\end{gathered}
$$

where $[a(s)]_{+}=\max \{a(s), 0\}$. Then $(C)$ holds.

For the special case $\int_{0}^{T} a(t) \sigma(h)(t) d t>0$ and $h \in$ $\widetilde{\mathbb{C}}(\mathbb{R} / T \mathbb{Z}):=\{h \in \mathbb{C}(\mathbb{R} / T \mathbb{Z}): \bar{h}=0\}$, one criterion has been developed by Cabada and Cid in [40]. 
Theorem 16 (see [40, Theorem 5.1]). Assume that $h \in$ $\widetilde{\mathbb{C}}(\mathbb{R} / T \mathbb{Z})$ and $\int_{0}^{T} a(t) \sigma(h)(t) d t>0$. Suppose further that there exists $1 \leq p \leq \infty$ such that

$$
(B(T))^{1+1 / q}\left\|\mathscr{A}_{+}\right\|_{p, T}<\mathbf{M}^{2}(2 q),
$$

where

$$
\begin{gathered}
B(T)=\int_{0}^{T} \sigma(-h)(t) d t, \\
\mathscr{A}_{+}(t)=a_{+}(t)(\sigma(h)(t))^{2-1 / p} .
\end{gathered}
$$

Then (C) holds.

Theorem 17 (see [35, Theorem 3.2]). Suppose that (25) satisfies (C) and

$$
\int_{0}^{T} a(t) \sigma(h)(t) d t>0
$$

Furthermore, assume that there exists a constant $r>0$ such that

$\left(G_{1}\right)$ there exists a continuous function $\phi_{r}>0$ such that $f(t, x, y) \geq \phi_{r}(t)$ for all $(t, x, y) \in[0, T] \times(0, r] \times$ $(-\infty, \infty)$

$\left(\mathrm{G}_{2}\right)$ there exist continuous, nonnegative functions $g(\cdot), h(\cdot)$, and $\varrho(\cdot)$ such that

$$
\begin{array}{r}
0 \leq f(t, x, y) \leq(g(x)+h(x)) \varrho(|y|), \\
\forall(t, x, y) \in[0, T] \times(0, r] \times \mathbb{R},
\end{array}
$$

where $g(\cdot)>0$ is nonincreasing, $h(\cdot) / g(\cdot)$ is nondecreasing in $(0, r]$, and $\varrho(\cdot)$ is non-decreasing in $(0, \infty)$,

$\left(\mathrm{G}_{3}\right)$ the following inequality holds:

$$
\frac{r}{g(\iota r)\{1+(h(r) / g(r))\} \varrho(L r)}>\omega^{*},
$$

where

$$
\begin{aligned}
& \omega(t)=\int_{0}^{T} G(t, s) d s, \quad L=\frac{2 \int_{0}^{T} a(t) \sigma(h)(t) d t}{\min _{0 \leq t \leq T} \sigma(h)(t)}, \\
& \iota=\frac{m}{M}, \quad m=\min _{0 \leq s, t \leq T} G(t, s), \quad M=\max _{0 \leq s, t \leq T} G(t, s),
\end{aligned}
$$

then (23) has at least one positive T-periodic solution $x$ with $0<\|x\| \leq r$.

Corollary 18 (see [35, Corollary 3.3]). Let the nonlinearity in (23) be

$$
f(t, x, y)=\left(1+|y|^{\gamma}\right)\left(x^{-\alpha}+\mu x^{\beta}\right)
$$

where $\alpha>0, \beta, \gamma \geq 0, \mu>0$ is a positive parameter. (i) If $\beta+\gamma<1$, then (23) has at least one positive periodic solution for each $\mu>0$.

(ii) If $\beta+\gamma \geq 1$, then (23) has at least one positive periodic solution for each $0<\mu<\mu_{1}^{*}$, where $\mu_{1}^{*}$ is some positive constant.

Corollary 19 (see [35, Corollary 3.4]). Let the nonlinearity in (23) be

$$
f(t, x, y)=\left(1+|y|^{\gamma}\right)\left(\frac{1}{x^{\alpha}}-\frac{\mu}{x^{\beta}}\right),
$$

where $\alpha>\beta>0, \gamma \geq 0$ with $\gamma<\alpha+1, \mu>0$ is a positive parameter. Then there exists a positive constant $\mu_{2}^{*}$ such that (23) has at least one positive T-periodic solution for each $0 \leq$ $\mu<\mu_{2}^{*}$.

Corollary 19 is interesting because the singularity on the right-hand side combines attractive and repulsive effects. The analysis of such differential equations with mixed singularities is at this moment very incomplete, and few references can be cited $[22,44]$. Therefore, the results in Corollary 19 can be regarded as one contribution to the literature trying to fill partially this gap in the study of singularities of mixed type.

As in the last section, if we assume that the linear equation (25)-(4) has a nonnegative Green's function, we can also get some results based on Schauder's fixed point theorem, and the results can cover the critical case.

\section{Singular Impulsive Differential Equations}

In this section, we will study the existence of periodic solutions for some singular differential equations with impulsive effects by using variational methods.

Firstly, we consider the following second-order nonautonomous singular problem:

$$
\begin{aligned}
& u^{\prime \prime}-\frac{b(t)}{u^{\alpha}}=e(t), \quad \text { a.e. } t \in(0, T), \\
& u(0)-u(T)=u^{\prime}(0)-u^{\prime}(T)=0,
\end{aligned}
$$

under the impulse conditions

$$
\Delta u^{\prime}\left(t_{j}\right)=I_{j}\left(u\left(t_{j}\right)\right), \quad j=1,2, \ldots, p-1,
$$

where $t_{j}, j=1,2, \ldots, p-1$ are the instants where the impulses occur and $0=t_{0}<t_{1}<t_{2}<\cdots<t_{p-1}<t_{p}=T, I_{j}: \mathbb{R} \rightarrow$ $\mathbb{R}(j=1,2, \ldots, p-1)$ are continuous.

Our result is presented as follows.

Theorem 20 (see [19, Theorem 1.1]). Assume that $\alpha>1$ and the following conditions hold.

$\left(S_{1}\right) b \in \mathbb{C}^{1}([0, T],(0, \infty))$ is $T$-periodic and $b^{\prime}(t) \geq 0$ for all $t \in[0, T]$.

$\left(S_{2}\right) e \in L^{2}([0, T], \mathbb{R})$ is $T$-periodic and $\int_{0}^{T} e(t) d t<0$.

$\left(S_{3}\right)$ There exist two constants $m, M$ such that for any $t \in \mathbb{R}$,

$$
m \leq I_{j}(t) \leq M, \quad j=1,2, \ldots, p-1,
$$

where $m<0$ and $0 \leq M<(-1 /(p-1)) \int_{0}^{T} e(t) d t$. 
$\left(S_{4}\right)$ For any $t \in \mathbb{R}$

$$
\int_{0}^{t} I_{j}(s) d s \geq 0, \quad j=1,2, \ldots, p-1 .
$$

Then problem (38)-(39) has at least one solution.

Remark 21. In fact, it is not difficult to find some functions $I_{j}$ satisfying $\left(S_{3}\right)$ and $\left(S_{4}\right)$. For example,

$$
I_{j}(t)=\sin t, \quad t \in \mathbb{R} .
$$

Let

$H_{T}^{1}=\{u:[0, T] \longrightarrow \mathbb{R} \mid u$ is absolutely continuous,

$$
\left.u(0)=u(T) \text { and } u^{\prime} \in L^{2}([0, T], \mathbb{R})\right\},
$$

with the inner product

$$
(u, v)=\int_{0}^{T} u(t) v(t) d t+\int_{0}^{T} u^{\prime}(t) v^{\prime}(t) d t, \quad \forall u, v \in H_{T}^{1}
$$

The corresponding norm is defined by

$$
\|u\|_{H_{\mathrm{T}}^{1}}=\left(\int_{0}^{T}|u(t)|^{2} d t+\int_{0}^{T}\left|u^{\prime}(t)\right|^{2} d t\right)^{1 / 2}, \quad \forall u \in H_{T}^{1} .
$$

Then $H_{T}^{1}$ is a Banach space (in fact it is a Hilbert space).

If $u \in H_{T}^{1}$, then $u$ is absolutely continuous and $u^{\prime} \in$ $L^{2}([0, T], \mathbb{R})$. In this case, $\Delta u^{\prime}(t)=u^{\prime}\left(t^{+}\right)-u^{\prime}\left(t^{-}\right)=0$ is not necessarily valid for every $t \in(0, T)$ and the derivative $u^{\prime}$ may exist some discontinuities. It may lead to impulse effects.

Following the ideas of [53], take $v \in H_{T}^{1}$ and multiply the two sides of the equality

$$
-u^{\prime \prime}+\frac{b(t)}{u^{\alpha}}+e(t)=0
$$

by $v$ and integrate from 0 to $T$, so we have

$$
\int_{0}^{T}\left[-u^{\prime \prime}+\frac{b(t)}{u^{\alpha}}+e(t)\right] v d t=0
$$

Note that since $u^{\prime}(0)-u^{\prime}(T)=0$, one has

$$
\begin{aligned}
\int_{0}^{T} u^{\prime \prime}(t) v(t) d t \\
=\sum_{j=0}^{p-1} \int_{t_{j}}^{t_{j+1}} u^{\prime \prime}(t) v(t) d t \\
=\sum_{j=0}^{p-1}\left(u^{\prime}\left(t_{j+1}^{-}\right) v\left(t_{j+1}^{-}\right)-u^{\prime}\left(t_{j}^{+}\right) v\left(t_{j}^{+}\right)\right) \\
\quad-\sum_{j=0}^{p-1} \int_{t_{j}}^{t_{j+1}} u^{\prime}(t) v^{\prime}(t) d t
\end{aligned}
$$

$$
\begin{aligned}
= & u^{\prime}(T) v(T)-u^{\prime}(0) v(0)-\sum_{j=1}^{p-1} \Delta u^{\prime}\left(t_{j}\right) v\left(t_{j}\right) \\
& -\int_{0}^{T} u^{\prime}(t) v^{\prime}(t) d t \\
= & -\sum_{j=1}^{p-1} I_{j}\left(u\left(t_{j}\right)\right) v\left(t_{j}\right)-\int_{0}^{T} u^{\prime}(t) v^{\prime}(t) d t .
\end{aligned}
$$

Combining with (47), we get

$$
\begin{aligned}
& \int_{0}^{T} u^{\prime}(t) v^{\prime}(t) d t+\sum_{j=1}^{p-1} I_{j}\left(u\left(t_{j}\right)\right) v\left(t_{j}\right) \\
& \quad+\int_{0}^{T} \frac{b(t)}{u^{\alpha}} v(t) d t+\int_{0}^{T} e(t) v(t) d t=0
\end{aligned}
$$

As a result, we introduce the following concept of a weak solution for problem (38)-(39).

Definition 22. One says that a function $u \in H_{T}^{1}$ is a weak solution of problem (38)-(39) if

$$
\begin{aligned}
& \int_{0}^{T} u^{\prime}(t) v^{\prime}(t) d t+\sum_{j=1}^{p-1} I_{j}\left(u\left(t_{j}\right)\right) v\left(t_{j}\right) \\
& +\int_{0}^{T} \frac{b(t)}{u^{\alpha}} v(t) d t+\int_{0}^{T} e(t) v(t) d t=0
\end{aligned}
$$

holds for any $v \in H_{T}^{1}$.

Define the functional $\Phi: H_{T}^{1} \rightarrow \mathbb{R}$ by

$$
\begin{aligned}
\Phi(u):= & \frac{1}{2} \int_{0}^{T}\left|u^{\prime}(t)\right|^{2} d t+\sum_{j=1}^{p-1} \int_{0}^{u\left(t_{j}\right)} I_{j}(s) d s \\
& +\int_{0}^{T} b(t)\left(\int_{1}^{u(t)} \frac{1}{s^{\alpha}} d s\right) d t+\int_{0}^{T} e(t) u(t) d t
\end{aligned}
$$

for every $u \in H_{T}^{1}$. Clearly, $\Phi_{\lambda}$ is well defined on $H_{T}^{1}$, continuously Gáteaux differentiable functional whose Gáteaux derivative is the functional $\Phi_{\lambda}^{\prime}(u)$, given by

$$
\begin{aligned}
\Phi_{\lambda}^{\prime}(u) v= & \int_{0}^{T} u^{\prime}(t) v^{\prime}(t) d t+\sum_{j=1}^{p-1} I_{j}\left(u\left(t_{j}\right)\right) v\left(t_{j}\right) \\
& -\int_{0}^{T} \frac{b(t)}{u^{\alpha}} v(t) d t+\int_{0}^{T} e(t) v(t) d t
\end{aligned}
$$

for any $v \in H_{T}^{1}$. Moreover, it is easy to verify that $\Phi_{\lambda}$ is weakly lower semicontinuous. Indeed, if $\left\{u_{n}\right\} \subset H_{T}^{1}, u \in H_{T}^{1}$, and $u_{n} \rightarrow u$, then $\left\{u_{n}\right\}$ converges uniformly to $u$ on $[0, T]$ 
and $u_{n} \rightarrow u$ on $L^{2}([0, T])$, and combining the fact that $\liminf \inf _{n \rightarrow \infty}\left\|u_{n}\right\|_{H_{T}^{1}} \geq\|u\|_{H_{T}^{1}}$, one has

$$
\begin{aligned}
& \liminf _{n \rightarrow \infty} \Phi_{\lambda}\left(u_{n}\right) \\
& =\liminf _{n \rightarrow \infty}\left(\frac{1}{2}\left\|u_{n}\right\|_{H_{T}^{1}}^{2}-\frac{1}{2} \int_{0}^{T}\left|u_{n}(t)\right|^{2} d t\right. \\
& +\sum_{j=1}^{p-1} \int_{0}^{u_{n}\left(t_{j}\right)} I_{j}(s) d s \\
& \quad-\int_{0}^{T} b(t)\left(\int_{1}^{u_{n}(t)} \frac{1}{s^{\alpha}} d s\right) d t \\
& \left.\quad+\int_{0}^{T} e(t) u_{n}(t) d t\right) \\
& \geq \frac{1}{2} \int_{0}^{T}\left|u^{\prime}(t)\right|^{2} d t+\sum_{j=1}^{p-1} \int_{0}^{u\left(t_{j}\right)} I_{j}(s) d s \\
& \quad-\int_{0}^{T} b(t)\left(\int_{1}^{u(t)} \frac{1}{s^{\alpha}} d s\right) d t+\int_{0}^{T} e(t) u(t) d t=\Phi_{\lambda}(u) .
\end{aligned}
$$

By the standard discussion, the critical points of $\Phi_{\lambda}$ are the weak solutions of problem (38)-(39), see $[53,54]$.

The following version of the mountain pass theorem will be used in our argument.

Theorem 23 (see [55, Theorem 4.10]). Let $X$ be a Banach space and let $\varphi \in C^{1}(X, \mathbb{R})$. Assume that there exist $x_{0}, x_{1} \in X$ and an open neighborhood $\Omega$ of $x_{0}$ such that $x_{1} \in X \backslash \bar{\Omega}$ and

$$
\max \left\{\varphi\left(x_{0}\right), \varphi\left(x_{1}\right)\right\}<\inf _{x \in \partial \Omega} \varphi(x) .
$$

Let

$$
\begin{gathered}
\Gamma=\left\{h \in C([0,1], X): h(0)=x_{0}, h(1)=x_{1}\right\}, \\
c=\inf _{h \in \Gamma \in[0,1]} \max _{s \in}(h(s)) .
\end{gathered}
$$

If $\varphi$ satisfies the (PS)-condition, that is, a sequence $\left\{u_{n}\right\}$ in $X$ satisfying $\varphi\left(u_{n}\right)$ is bounded and $\varphi^{\prime}\left(u_{n}\right) \rightarrow 0$ as $n \rightarrow \infty$ has a convergent subsequence, then $c$ is a critical value of $\varphi$ and $c>\max \left\{\varphi\left(x_{0}\right), \varphi\left(x_{1}\right)\right\}$.

Next we consider $T$-periodic solution for another impulsive singular problem:

$$
u^{\prime \prime}(t)-\frac{1}{u^{\alpha}(t)}=e(t)
$$

under impulsive conditions

$$
\Delta u^{\prime}\left(t_{j}\right)=I_{j}\left(u\left(t_{j}\right)\right), \quad j=1,2, \ldots, p-1,
$$

where $\alpha \geq 1$, e $\in L^{1}([0, T], \mathbb{R})$ is $T$-periodic, $\Delta u^{\prime}\left(t_{j}\right)=$ $u^{\prime}\left(t_{j}^{+}\right)-u^{\prime}\left(t_{j}^{-}\right)$with $u^{\prime}\left(t_{j}^{ \pm}\right)=\lim _{t \rightarrow t_{j}^{ \pm}} u^{\prime}(t) ; t_{j}, j=1,2, \ldots, p-1$ are the instants where the impulses occur, and $0=t_{0}<t_{1}<$ $t_{2}<\cdots<t_{p-1}<t_{p}=T, t_{j+p}=t_{j}+T ; I_{j}: \mathbb{R} \rightarrow \mathbb{R}(j=$ $1,2, \ldots, p-1)$ are continuous and $I_{j+p} \equiv I_{j}$.

In 1987, Lazer and Solimini [29] proved a famous result as follows.

Theorem 24 (see [29]). Assume that $e \in L^{1}([0, T], \mathbb{R})$ is $T$ periodic. Then problem (56) has a positive T-periodic weak solution if and only if $\int_{0}^{T} e(t) d t<0$.

From Theorem 24, if $\int_{0}^{T} e(t) d t \geq 0$, then problem (52) does not have a positive $T$-periodic weak solution. However, if the impulses happen, for this singular problem may exist a positive $T$-periodic weak solution. Inspired by the above facts, our aim is to reveal a new existence result on positive $T$ periodic solution for singular problem (56) when impulsive effects are considered, that is, problem (56)-(57). Indeed, this periodic solution is generated by impulses. Here, we say a solution is generated by impulses if this solution is nontrivial when $I_{j} \not \equiv 0$ for some $1<j<p-1$, but it is trivial when $I_{j} \equiv 0$ for all $1<j<p-1$. For example, if problem (56)-(57) does not possess positive periodic solution when $I_{j} \equiv 0$ for all $1<j<p-1$, then a positive periodic solution $u$ of problem (56)-(57) with $I_{j} \not \equiv 0$ for some $1<j<p-1$ is called a positive periodic solution generated by impulses.

Our result is presented as follows.

Theorem 25 (see [35, Theorem 1.2]). Assume the following:

$\left(S_{1}\right) e \in L^{1}([0, T], \mathbb{R})$ is $T$-periodic and $\int_{0}^{T} e(t) d t \geq 0$;

$\left(S_{2}\right)$ there exist two constants $m, M$ such that for any $s \in \mathbb{R}$,

$$
m \leq I_{j}(s) \leq M, \quad j=1,2, \ldots, p-1,
$$

where $m \leq M<(-1 /(p-1)) \int_{0}^{T} e(t) d t \leq 0$.

Then problem (56)-(57) has at least a positive $T$-periodic solution.

\section{Singular Differential Systems}

In this section, we will consider the system of Hill's equations

$$
\begin{array}{r}
u_{i}^{\prime \prime}(t)+a_{i}(t) u_{i}(t)=F_{i}\left(t, u_{1}(t), u_{2}(t), \ldots, u_{n}(t)\right), \\
1 \leq i \leq n .
\end{array}
$$

Here, $a_{i}$ and $F_{i}$ are $T$-periodic in the variable $t, a_{i} \in L^{1}[0, T]$, and the nonlinearities $F_{i}\left(t, x_{1}, x_{2}, \ldots, x_{n}\right)$ can be singular at $x_{j}=0$ where $j \in\{1,2, \ldots, n\}$.

Throughout, let $u=\left(u_{1}, u_{2}, \ldots, u_{n}\right)$. We are interested in establishing the existence of continuous $T$-periodic solutions $u$ of the system (59), that is, $u \in(C(\mathbb{R}))^{n}$ and $u(t)=u(t+T)$ for all $t \in \mathbb{R}$. Moreover, we are concerned with constant-sign solutions $u$, by which we mean $\theta_{i} u_{i}(t) \geq 0$ for all $t \in \mathbb{R}$ and $1 \leq i \leq n$, where $\theta_{i} \in\{1,-1\}$ is fixed. Note that positive solution, the usual consideration in the literature, is a special case of constant-sign solution when $\theta_{i}=1$ for $1 \leq i \leq n$. 
We will employ the Schauder's fixed point theorem to establish the existence of solutions. Indeed, in Section 5.1 we will first tackle a particular case of (59) when

$$
F_{i}(t, u(t))=\partial_{2} h_{i}\left(t, \frac{1}{2}|u(t)|^{2}\right) u_{i}(t)+f_{i}(t) .
$$

Here, $\partial_{2} h_{i}$ is the partial derivative of $h_{i}$ with respect to the second variable, and $|\cdot|$ is a norm in $\mathbb{R}^{n}$. The particular case (60) occurs in the problem [36]

$$
\ddot{u}(t)+\nabla_{u} P(t, u(t))=f(t),
$$

where the potential

$$
P(t, u)=\frac{1}{2} a(t)|u|^{2}-h\left(t, \frac{1}{2}|u|^{2}\right),
$$

and $h$ presents a singularity of the repulsive type, that is, $\lim _{|x| \rightarrow 0} h(t, x)=\infty$ uniformly in $t$. The general problem (59) will be investigated in Section 5.2; here the singularities are not necessarily generated by a potential as in the case of (60). To illustrate our results, several examples will be presented.

In [45], the authors use a nonlinear alternative of the Leray-Schauder type and a fixed point theorem in cones to establish the existence of two positive periodic solutions for the system

$$
\ddot{u}(t)+a(t) u(t)=G(u(t)),
$$

where $G$ can be expressed as a sum of two positive functions satisfying certain monotone conditions. Therefore, the results in [45] are not applicable to (59) with $F_{i}$ as in (60). In [45] it is also shown that the system

$$
\begin{aligned}
& u_{1}^{\prime \prime}(t)+a_{1}(t) u_{1}(t)=\left(\sqrt{u_{1}^{2}+u_{2}^{2}}\right)^{-\beta}+v\left(\sqrt{u_{1}^{2}+u_{2}^{2}}\right)^{\gamma} \\
& u_{2}^{\prime \prime}(t)+a_{2}(t) u_{2}(t)=\left(\sqrt{u_{1}^{2}+u_{2}^{2}}\right)^{-\beta}+v\left(\sqrt{u_{1}^{2}+u_{2}^{2}}\right)^{\gamma}
\end{aligned}
$$

has a solution when $\beta>0, \gamma \in[0,1)$, and $\nu>0$. We will generalize the system (64) in Examples 46-48 to allow $v$ to be zero or negative. The improvement is possible probably due to the fact that we do not need to make a technical truncation to get compactness when we employ the Schauder fixed point theorem as compared to when the Leray-Schauder alternative is used. In fact, the set that we work on excludes the singularities. The results presented in this section not only generalize the papers $[36,39,45]$ to systems and existence of constant-sign solutions, but also improve and/or complement the results in these earlier work as well as other research papers [56-60]. This section is based on the work in [61].

5.1. Existence Results for (60). In this section we will consider the system of Hill's equations

$$
\begin{array}{r}
u_{i}^{\prime \prime}(t)+a_{i}(t) u_{i}(t)=\partial_{2} h_{i}\left(t, \frac{1}{2}|u(t)|^{2}\right) u_{i}(t)+f_{i}(t), \\
1 \leq i \leq n .
\end{array}
$$

Here, $\partial_{2} h_{i}(t, s) \equiv(\partial / \partial s) h_{i}(t, s)$ and $|\cdot|$ is a norm in $\mathbb{R}^{n}$. Moreover, $a_{i}(t), \partial_{2} h_{i}(t, s)$, and $f_{i}(t)$ are $T$-periodic in $t, a_{i} \in$ $L^{1}[0, T], f_{i} \in L^{1}[0, T]$, and $\partial_{2} h_{i}(t, s)$ can be singular at $s=0$.

To seek a $T$-periodic solution $u^{T}=\left(u_{1}^{T}, u_{2}^{T}, \ldots, u_{n}^{T}\right)$ of the system $(65)$, we first obtain a solution $u^{*}=\left(u_{1}^{*}, u_{2}^{*}, \ldots, u_{n}^{*}\right)$ of the following system of boundary value problems:

$$
\begin{aligned}
& u_{i}^{\prime \prime}(t)+a_{i}(t) u_{i}(t) \\
&= \partial_{2} h_{i}\left(t, \frac{1}{2}|u(t)|^{2}\right) u_{i}(t)+f_{i}(t), \quad t \in[0, T], \\
& u_{i}(0)=u_{i}(T), \quad u_{i}^{\prime}(0)=u_{i}^{\prime}(T), \quad 1 \leq i \leq n .
\end{aligned}
$$

Then, set

$$
u^{T}(t)=u^{*}(t-m T), \quad t \in[m T,(m+1) T], m \in \mathbb{Z} .
$$

Our main tool is Schauder's fixed point theorem, which is stated below for completeness.

Theorem 26 (see [62]). Let $\Omega$ be a convex subset of a Banach space $B$ and $S: \Omega \rightarrow \Omega$ a continuous and compact map. Then $S$ has a fixed point.

To begin, let $g_{i}$ be Green's function of the boundary value problem

$$
\begin{aligned}
& x^{\prime \prime}(t)+a_{i}(t) x(t)=0, \quad t \in[0, T], \\
& x(0)=x(T), \quad x^{\prime}(0)=x^{\prime}(T) .
\end{aligned}
$$

Throughout, we will assume that the functions $a_{i} \in L^{1}[0, T]$ are such that

(C1) the Hill's equation $x^{\prime \prime}(t)+a_{i}(t) x(t)=0$ is nonresonant (i.e., the unique periodic solution is the trivial solution), and $g_{i}(t, s) \geq 0$ for all $(t, s) \in[0, T] \times[0, T]$.

Note that Torres [46] has a result on $a_{i}(t)$ that ensures that condition (C1) is satisfied. In fact, if $a_{i}(t)=k^{2}$, then $(\mathrm{C} 1)$ holds if $k \in(0, \pi / T]$; if $a_{i}(t)$ is not a constant, then $(\mathrm{C} 1)$ is valid if the $L_{p}$ norm of $a_{i}(t)$ is bounded above by some specific constant.

Let $\theta_{i} \in\{1,-1\}, 1 \leq i \leq n$ be fixed. Define

$$
\phi_{i}(t)=\int_{0}^{T} g_{i}(t, s) \theta_{i} f_{i}(s) d s, \quad t \in[0, T], 1 \leq i \leq n
$$

We also let

$$
\phi_{i}^{\min }=\min _{t \in[0, T]} \phi_{i}(t), \quad \phi_{i}^{\max }=\max _{t \in[0, T]} \phi_{i}(t) .
$$

We now present our main result which tackles (65) when the norm $|\cdot|$ in $\mathbb{R}^{n}$ is the $l_{p}$ norm or the $l_{\infty}$ norm.

Theorem 27. Assume that the following conditions hold for each $1 \leq i \leq n$ : $(C 1)$,

$$
\text { (C2) } \phi_{i}^{\min }>0 \text {; }
$$


(C3) let $H_{i}(t, s)=(\partial / \partial s) h_{i}(t, s)$; for any numbers $b, b^{\prime}$ with $b^{\prime} \geq b>0$, the function $H_{i}:[0, T] \times\left[b, b^{\prime}\right] \rightarrow \mathbb{R}$ is an $L^{1}$-Carathéodory function, that is,

(i) the map $s \mapsto H_{i}(t, s)$ is continuous for almost all $t \in[0, T]$,

(ii) the map $t \mapsto H_{i}(t, s)$ is measurable for all $s \in$ $\left[b, b^{\prime}\right]$,

(iii) for any $r>0$, there exists $\mu_{r, i} \in L^{1}[0, T]$ such that $|s| \leq r\left(s \in\left[b, b^{\prime}\right]\right)$ implies $\left|H_{i}(t, s)\right| \leq \mu_{r, i}(t)$ for almost all $t \in[0, T]$;

(C4) $(\partial / \partial s) h_{i}(t, s) \geq 0$ for $t \in[0, T]$ and $s>0$;

(C5) there exist $c_{i}>0$ and $\alpha_{i}>0$ such that

$$
\frac{\partial}{\partial s} h_{i}(t, s) \leq c_{i} s^{-\alpha_{i}}, \quad t \in[0, T], s>0
$$

(C6) the norm $|\cdot|$ is the $l_{p}$ norm where $1 \leq p \leq \infty$ is fixed, and

$$
\int_{0}^{T} g_{i}(t, s) d s<A_{i}^{p}\left(c_{i} 2^{\alpha_{i}}\right)^{-1}, \quad t \in[0, T],
$$

where

$$
A_{i}^{p}= \begin{cases}{\left[\sum_{k=1}^{n}\left(\phi_{k}^{\min }\right)^{p}\right]^{2 \alpha_{i} / p},} & 1 \leq p<\infty, \\ {\left[\max _{1 \leq k \leq n} \phi_{k}^{\min }\right]^{2 \alpha_{i}},} & p=\infty .\end{cases}
$$

Then, (65) has a T-periodic constant-sign solution $u^{T} \in$ $(C(\mathbb{R}))^{n}$ such that

$$
\phi_{i}^{\min } \leq \theta_{i} u_{i}^{T}(t) \leq R_{i}, \quad t \in \mathbb{R}, 1 \leq i \leq n,
$$

where

$$
\begin{array}{r}
R_{i} \geq \phi_{i}^{\min }, \quad R_{i} \geq \phi_{i}^{\max }\left[1-\frac{c_{i} 2^{\alpha_{i}}}{A_{i}^{p}} \max _{t \in[0, T]} \int_{0}^{T} g_{i}(t, s) d s\right]^{-1}, \\
1 \leq i \leq n .
\end{array}
$$

Theorem 27 is proved using Theorem 26; in fact we will seek a constant-sign solution of $(66)$ in $(C[0, T])^{n}$ and then extend it to a $T$-periodic constant-sign solution of (65) as in (67). Here, let $\Omega$ be the closed convex set given by

$$
\begin{gathered}
\Omega=\left\{u \in(C[0, T])^{n} \mid \phi_{i}^{\min } \leq \theta_{i} u_{i}(t) \leq R_{i},\right. \\
\left.t \in[0, T], 1 \leq i \leq n \mid \phi_{i}^{\min }\right\},
\end{gathered}
$$

where $R_{i}\left(\geq \phi_{i}^{\min }>0\right)$ is chosen as in (75), and define the operator $S: \Omega \rightarrow(C[0, T])^{n}$ as

$$
S u(t)=\left(S_{1} u(t), S_{2} u(t), \ldots, S_{n} u(t)\right), \quad t \in[0, T],
$$

where

$$
\begin{array}{r}
S_{i} u(t)=\int_{0}^{T} g_{i}(t, s)\left[\partial_{2} h_{i}\left(s, \frac{1}{2}|u(s)|^{2}\right) u_{i}(s)+f_{i}(s)\right] d s, \\
t \in[0, T], 1 \leq i \leq n .
\end{array}
$$

Clearly, a fixed point of $S u=u$ is a solution of (66). We can show that $S(\Omega) \subseteq \Omega$; that is, $S_{i}(\Omega) \subseteq \Omega$ for each $1 \leq i \leq n$. Further, we can prove that $S: \Omega \rightarrow \Omega$ is continuous and compact; that is, $S_{i} u$ is bounded and is equicontinuous for any $u \in \Omega$ and $1 \leq i \leq n$. By Theorem 26, the system (66) has a constant-sign solution $u^{*} \in \Omega$. Now, a $T$-periodic constantsign solution $u^{T}$ of (65) can be obtained as in (67).

Remark 28. The constants $c_{i}$ that appear in (C5) determine the upper bounds $R_{i}$ of the solution $u_{i}^{T}, 1 \leq i \leq n$. Noting (75), we see that a smaller (bigger) $c_{i}$ gives a smaller (bigger) $R_{i}$, and hence a smaller (bigger) set $\Omega$ where the solution lies.

In the next result, we will relax the condition (C6). The tradeoff is the upper bounds $R_{i}$ of the solution that may be bigger than those in (75). Also the bounds $R_{i}$ do not depend on $p$ ( $p$ as in $l_{p}$ norm) and so the information of $p$ is not utilized. This result is obtained by following the main arguments in the derivation of Theorem 27 but modify the proof of $\theta_{i} S_{i} u(t) \leq R_{i}, t \in[0, T]$.

Theorem 29. Assume that (C1)-(C5) hold for each $1 \leq i \leq n$. The norm $|\cdot|$ is the $l_{p}$ norm where $1 \leq p \leq \infty$ is fixed. Then (65) has a $T$-periodic constant-sign solution $u^{T} \in(C(\mathbb{R}))^{n}$ such that

$$
\phi_{i}^{\min } \leq \theta_{i} u_{i}^{T}(t) \leq R_{i}, \quad t \in \mathbb{R}, 1 \leq i \leq n,
$$

where, for $1 \leq i \leq n$ we have $R_{i} \geq \phi_{i}^{\min }$,

$$
\begin{array}{r}
R_{i}^{2 \alpha_{i}}>c_{i} 2^{\alpha_{i}} \max _{t \in[0, T]} \int_{0}^{T} g_{i}(t, s) d s, \quad \text { if } \alpha_{i} \in\left(0, \frac{1}{2}\right), \\
R_{i}\left[1-c_{i} 2^{\alpha_{i}} R_{i}^{-2 \alpha_{i}} \max _{t \in[0, T]} \int_{0}^{T} g_{i}(t, s) d s\right] \geq \phi_{i}^{\max }, \\
\text { if } \alpha_{i} \in\left(0, \frac{1}{2}\right), \\
R_{i} \geq c_{i} 2^{\alpha_{i}}\left(\phi_{i}^{\min }\right)^{1-2 \alpha_{i}} \max _{t \in[0, T]} \int_{0}^{T} g_{i}(t, s) d s+\phi_{i}^{\max }, \\
\text { if } \alpha_{i} \geq \frac{1}{2} .
\end{array}
$$

Remark 30. A similar remark as Remark 28 also holds for Theorem 29. Moreover, we note that the upper bounds $R_{i}$ that fulfill (80)-(82) are independent of $p$, thus the information of $|\cdot|$ being a particular $l_{p}$ norm is not used. On the other hand, in Theorem 27, the upper bounds $R_{i}$ that satisfy (75) depend on $p$. The sharpness of the bounds in both theorems cannot be compared in general; however, we will give an example at the end of this section to illustrate the results. 
In the next result, we will relax the condition (C2). Here, we allow $\phi_{i}(t) \leq 0$ for some $i \in\{1,2, \ldots, n\}$ and some $t \in$ $[0, T]$.

\section{Theorem 31. Suppose that}

(C7) there exists $j \in\{1,2, \ldots, n\}$ such that $\phi_{j}^{\min }>0$.

Let $J=\left\{j \in\{1,2, \ldots, n\} \mid \phi_{j}^{\min }>0\right\}$ and let $J^{\prime}=\{1,2, \ldots, n\} \backslash J$. Assume that the following conditions hold for each $1 \leq i \leq n$ : (C1), (C3), (C4), and

(C8) there exist $c_{i}>0$ such that

$$
\frac{\partial}{\partial s} h_{i}(t, s) \leq c_{i} s^{-\alpha_{i}}, \quad t \in[0, T], s>0
$$

where $\alpha_{j}>0$ for $j \in J$ and $\alpha_{k} \in(0,1 / 2)$ for $k \in J^{\prime}$.

Further, let the following hold for each $j \in J$ :

(C9) the norm $|\cdot|$ is the $l_{p}$ norm where $1 \leq p \leq \infty$ is fixed, and

$$
\int_{0}^{T} g_{j}(t, s) d s<\bar{A}_{j}^{p}\left(c_{j} 2^{\alpha_{j}}\right)^{-1}, \quad t \in[0, T],
$$

where

$$
\bar{A}_{j}^{p}= \begin{cases}{\left[\sum_{\ell \in J}\left(\phi_{\ell}^{\min }\right)^{p}\right]^{2 \alpha_{j} / p},} & 1 \leq p<\infty, \\ {\left[\max _{\ell \in J} \phi_{\ell}^{\min }\right]^{2 \alpha_{j}},} & p=\infty\end{cases}
$$

Then, (65) has a T-periodic solution $u^{T} \in(C(\mathbb{R}))^{n}$ such that

$$
\begin{gathered}
\phi_{j}^{\min } \leq \theta_{j} u_{j}^{T}(t) \leq R_{j}, \quad t \in \mathbb{R}, j \in J, \\
\left|u_{k}^{T}(t)\right| \leq R_{k}, \quad t \in \mathbb{R}, k \in J^{\prime},
\end{gathered}
$$

where

$$
\begin{gathered}
R_{j} \geq \phi_{j}^{\min } \\
R_{j} \geq \phi_{j}^{\max }\left[1-c_{j} 2^{\alpha_{j}}\left(\bar{A}_{j}^{p}\right)^{-1} \max _{t \in[0, T]} \int_{0}^{T} g_{j}(t, s) d s\right]^{-1}, \quad j \in J, \\
R_{k}^{2 \alpha_{k}}>c_{k} 2^{\alpha_{k}} \max _{t \in[0, T]} \int_{0}^{T} g_{k}(t, s) d s, \quad k \in J^{\prime}, \\
R_{k}\left[1-c_{k} 2^{\alpha_{k}} R_{k}^{-2 \alpha_{k}} \max _{t \in[0, T]} \int_{0}^{T} g_{k}(t, s) d s\right] \geq \max _{t \in[0, T]}\left|\phi_{k}(t)\right|, \\
k \in J^{\prime} .
\end{gathered}
$$

To derive Theorem 31, we let the closed convex set $\Omega^{*}$ be $\Omega^{*}=\left\{u \in(C[0, T])^{n} \mid \phi_{j}^{\min } \leq \theta_{j} u_{j}(t) \leq R_{j}, t \in[0, T]\right.$,

$$
\left.j \in J ;\left|u_{k}(t)\right| \leq R_{k}, t \in[0, T], k \in J^{\prime}\right\},
$$

where $R_{j}\left(\geq \phi_{j}^{\text {min }}>0\right.$ ) and $R_{k}$ are chosen as in (87)-(89). Next, we define the operator $S: \Omega^{*} \rightarrow(C[0, T])^{n}$ as in (78) and show that Theorem 26 is applicable.

Remark 32. From the conclusion of Theorem 29, we see that the solution $u^{T}$ is "partially" of constant sign, in the sense that $\theta_{j} u_{j}^{T}(t) \geq 0$ for $j \in J$, but may not be so for $j \in J^{\prime}$. Further, the constants $c_{i}$ that appear in (C8) determine the upper bounds $R_{i}$ of the solution $u_{i}^{T}, 1 \leq i \leq n$. From (87) and (88), we see that a smaller (bigger) $c_{i}$ gives a smaller (bigger) $R_{i}$, and hence a smaller (bigger) set $\Omega^{*}$ where the solution lies.

Using similar arguments as in the derivation of Theorems 31 and 29 (in getting $S_{j} u \in \Omega^{*}$ for $j \in J$ and $u \in \Omega^{*}$ ), we obtain the following result.

Theorem 33. Suppose that (C7) hold. Let $J=\{j \in$ $\left.\{1,2, \ldots, n\} \mid \phi_{j}^{\mathrm{min}}>0\right\}$ and let $J^{\prime}=\{1,2, \ldots, n\} \backslash J$. Assume the following conditions hold for each $1 \leq i \leq n$ : (C1), (C3), (C4), and (C8). Then, (65) has a T-periodic solution $u^{T} \in(C(\mathbb{R}))^{n}$ such that

$$
\begin{gathered}
\phi_{j}^{\min } \leq \theta_{j} u_{j}^{T}(t) \leq R_{j}, \quad t \in \mathbb{R}, j \in J, \\
\left|u_{k}^{T}(t)\right| \leq R_{k}, \quad t \in \mathbb{R}, k \in J^{\prime},
\end{gathered}
$$

where

$$
\begin{gathered}
R_{j} \geq \phi_{j}^{\min }, \quad j \in J, \\
R_{j}^{2 \alpha_{j}}>c_{j} 2^{\alpha_{j}} \max _{t \in[0, T]} \int_{0}^{T} g_{j}(t, s) d s, \quad \text { if } \alpha_{j} \in\left(0, \frac{1}{2}\right), \quad j \in J, \\
R_{j}\left[1-c_{j} 2^{\alpha_{j}} R_{j}^{-2 \alpha_{j}} \max _{t \in[0, T]} \int_{0}^{T} g_{j}(t, s) d s\right] \geq \phi_{j}^{\max }, \\
R_{j} \geq c_{j} 2^{\alpha_{j}}\left(\phi_{j}^{\min }\right)^{1-2 \alpha_{j}} \max _{t \in[0, T]} \int_{0}^{T} g_{j}(t, s) d s+\phi_{j}^{\max }, \\
\text { if } \alpha_{j} \geq \frac{1}{2}, j \in J, \\
R_{k}^{2 \alpha_{k}}>c_{k} 2^{\alpha_{k}} \max _{t \in[0, T]} \int_{0}^{T} g_{k}(t, s) d s, \quad k \in J^{\prime}, \\
R_{k}\left[1-c_{k} 2^{\alpha_{k}} R_{k}^{-2 \alpha_{k}} \max _{t \in[0, T]} \int_{0}^{T} g_{k}(t, s) d s\right] \geq \max _{t \in[0, T]}\left|\phi_{k}(t)\right|, \\
k \in J^{\prime} .
\end{gathered}
$$

Remark 34. A similar remark as Remark 32 holds for Theorem 33. Also, we observe once again that the upper bounds $R_{j}$ that fulfill (92) are independent of $p$, thus the information of $|\cdot|$ being a particular $l_{p}$ norm is not used. On the other hand, in Theorem 31, the upper bounds $R_{j}$ that satisfy (87) depend on $p$. 
We will now present an example that illustrates Theorems 27 and 29.

Example 35. Consider (65) when

$$
\begin{gathered}
T=2 \pi, \quad n=2, \quad a_{1}(t)=a_{2}(t)=\frac{1}{4}, \\
f_{1}(t)=1, \quad f_{2}(t)=\frac{1}{2}, \quad h_{1}(t, s)=\frac{\ln (s+1)}{|\sin t|+1}, \\
h_{2}(t, s)=\frac{\ln (s+1)}{3(|\cos t|+1)}, \quad|\cdot|=l_{p} \operatorname{norm}(1 \leq p \leq \infty) .
\end{gathered}
$$

Fix $\theta_{i}=1,1 \leq i \leq n$, that is, we are seeking positive solutions. The corresponding Green's function has the explicit expression [36]

$$
g_{1}(t, s)=g_{2}(t, s)= \begin{cases}\cos \frac{1}{2}(t-s-\pi), & 0 \leq s \leq t \leq 2 \pi \\ \cos \frac{1}{2}(s-t-\pi), & 0 \leq t \leq s \leq 2 \pi\end{cases}
$$

Condition $(\mathrm{C} 1)$ is satisfied. By direct computation, we get $\phi_{1}(t)=4$ and $\phi_{2}(t)=2$ for $t \in[0,2 \pi]$. Thus, (C2) is fulfilled with

$$
\phi_{1}^{\min }=\phi_{1}^{\max }=4, \quad \phi_{2}^{\min }=\phi_{2}^{\max }=2 .
$$

Moreover, we have

$$
\begin{gathered}
\frac{\partial}{\partial s} h_{1}(t, s)=\frac{1}{|\sin t|+1} \frac{1}{s+1} \leq \frac{1}{s+1} \leq \frac{1}{s} \\
\frac{\partial}{\partial s} h_{2}(t, s)=\frac{1}{3(|\cos t|+1)} \frac{1}{s+1} \leq \frac{1}{3(s+1)} \leq \frac{1}{3 s}
\end{gathered}
$$

and so it is clear that (C4) and (C5) are satisfied with

$$
\alpha_{1}=1, \quad c_{1}=1, \quad \alpha_{2}=1, \quad c_{2}=\frac{1}{3} .
$$

Finally, we compute

$$
\begin{gathered}
A_{1}^{p}=\mathrm{A}_{2}^{p}=\left(4^{p}+2^{p}\right)^{2 / p}, \quad 1 \leq p<\infty, \\
A_{1}^{\infty}=A_{2}^{\infty}=16 .
\end{gathered}
$$

Since $\int_{0}^{2 \pi} g_{i}(t, s) d s=4$ for $t \in[0,2 \pi]$ and $i=1,2$, we check that (C6) holds for all $1 \leq p \leq \infty$.

All the conditions of Theorem 27 are satisfied, thus we conclude that the problem (65) with (94) has a positive $2 \pi$ periodic solution $u=\left(u_{1}, u_{2}\right)$ such that

$$
\phi_{i}^{\min } \leq u_{i}(t) \leq R_{i}, \quad t \in \mathbb{R}, i=1,2,
$$

where (from (75))

$$
R_{i} \geq \phi_{i}^{\max }\left[1-\frac{8 c_{i}}{A_{i}^{p}}\right]^{-1} \equiv L_{i}^{p}, \quad 1 \leq p \leq \infty, i=1,2 .
$$

We can also apply Theorem 29 to conclude that the problem (65) with (94) has a positive $2 \pi$-periodic solution $u=\left(u_{1}, u_{2}\right)$ satisfying (100) and (from (82))

$$
R_{i} \geq 8 c_{i}\left(\phi_{i}^{\min }\right)^{-1}+\phi_{i}^{\max } \equiv M_{i}, \quad i=1,2 .
$$

As mentioned in Remark 30, in general we cannot compare $L_{i}^{p}$ and $M_{i}$. In fact, a direct calculation gives

$$
\begin{aligned}
& p=1: \\
& L_{1}^{1}=5.14<M_{1}=6, \quad L_{2}^{1}=2.16<M_{2}=3.33, \\
& p=2: \\
& L_{1}^{2}=6.67>M_{1}=6, \quad L_{2}^{2}=2.31<M_{2}=3.33, \\
& p=\infty: \\
& L_{1}^{\infty}=8>M_{1}=6, \quad L_{2}^{\infty}=2.4<M_{2}=3.33 .
\end{aligned}
$$

5.2. Existence Results for (59). In this section we will consider the general system of Hill's equations

$$
u_{i}^{\prime \prime}(t)+a_{i}(t) u_{i}(t)=F_{i}(t, u(t)), \quad 1 \leq i \leq n .
$$

Here, $a_{i}$ and $F_{i}$ are $T$-periodic in the variable $t, a_{i} \in L^{1}[0, T]$, and the nonlinearities $F_{i}\left(t, x_{1}, x_{2}, \ldots, x_{n}\right)$ can be singular at $x_{j}=0$ where $j \in\{1,2, \ldots, n\}$.

Once again, to obtain a $T$-periodic solution $u^{T}=$ $\left(u_{1}^{T}, u_{2}^{T}, \ldots, u_{n}^{T}\right)$ of the system (104), we first seek a solution $u^{*}=\left(u_{1}^{*}, u_{2}^{*}, \ldots, u_{n}^{*}\right)$ of the following system of boundary value problems:

$$
\begin{array}{ll}
u_{i}^{\prime \prime}(t)+a_{i}(t) u_{i}(t)=F_{i}(t, u(t)), & t \in[0, T], \\
u_{i}(0)=u_{i}(T), & u_{i}^{\prime}(0)=u_{i}^{\prime}(T), \quad 1 \leq i \leq n .
\end{array}
$$

The periodic solution is then given by

$$
u^{T}(t)=u^{*}(t-m T), \quad t \in[m T,(m+1) T], m \in \mathbb{Z} .
$$

With $g_{i}$ being the Green's function of the boundary value problem (68), throughout we will assume that $(\mathrm{C} 1)$ is satisfied. Moreover, for fixed $\theta_{i} \in\{-1,1\}$ and $T$-periodic functions $q_{i} \in$ $L^{1}[0, T], 1 \leq i \leq n$, we define

$$
\eta_{i}(t)=\int_{0}^{T} g_{i}(t, s) \theta_{i} q_{i}(s) d s, \quad t \in[0, T], 1 \leq i \leq n
$$

and also

$$
\eta_{i}^{\min }=\min _{t \in[0, T]} \eta_{i}(t), \quad \eta_{i}^{\max }=\max _{t \in[0, T]} \eta_{i}(t) .
$$

For $b \geq b^{\prime} \geq 0$ and $1 \leq i \leq n$, we denote the interval

$$
\left[b, b^{\prime}\right]_{i}= \begin{cases}{\left[b, b^{\prime}\right],} & \text { if } \theta_{i}=1 \\ {\left[-b^{\prime},-b\right],} & \text { if } \theta_{i}=-1\end{cases}
$$

A similar definition is valid for $\left(b, b^{\prime}\right)_{i}$.

Using Schauder's fixed point theorem, we will establish existence results for the system (104). 
Theorem 36. Assume the following conditions hold for each $1 \leq i \leq n:(C 1)$;

(C10) for any numbers $b_{j}, b_{j}^{\prime}, 1 \leq j \leq n$ with $b_{j}^{\prime} \geq b_{j}>0$, the function $F_{i}:[0, T] \times \prod_{j=1}^{n}\left[b_{j}, b_{j}^{\prime}\right]_{j} \rightarrow \mathbb{R}$ is a $L^{1}$ Carathéodory function, that is,

(i) the map $u \mapsto F_{i}(t, u)$ is continuous for almost all $t \in[0, T]$

(ii) the map $t \mapsto F_{i}(t, u)$ is measurable for all $u \in$ $\prod_{j=1}^{n}\left[b_{j}, b_{j}^{\prime}\right]_{j}$,

(iii) for any $r>0$, there exists $\mu_{r, i} \in L^{1}[0, T]$ such that $|u| \leq r\left(u \in \prod_{j=1}^{n}\left[b_{j}, b_{j}^{\prime}\right]_{j}\right)$ implies $\left|F_{i}(t, u)\right| \leq$ $\mu_{r, i}(t)$ for almost all $t \in[0, T]$;

(C11) there exist $\beta_{i}>0, \gamma_{i} \in[0,1)$, and T-periodic functions $w_{i}, q_{i}$ with $w_{i} \in L^{1}[0, T], q_{i} \in L^{1}[0, T]$ and $w_{i}(t)>0$ for a.e. $t \in[0, T]$ such that

$$
\begin{array}{r}
\theta_{i} q_{i}(t)|u|^{\gamma_{i}} \leq \theta_{i} F_{i}(t, u) \leq \theta_{i} q_{i}(t)|u|^{\gamma_{i}}+w_{i}(t)|u|^{-\beta_{i}}, \\
t \in[0, T], u \in \prod_{k=1}^{n}(0, \infty)_{k}
\end{array}
$$

(here $|\cdot|$ is the $l_{p}$ norm where $1 \leq p \leq \infty$ is fixed);

(C12) $\eta_{i}^{\min }>0$.

Then, (104) has a T-periodic constant-sign solution $u^{T} \in$ $(C(\mathbb{R}))^{n}$ such that

$$
r_{i} \leq \theta_{i} u_{i}^{T}(t) \leq R_{i}, \quad t \in \mathbb{R}, 1 \leq i \leq n,
$$

where, for $1 \leq i \leq n$ one has

$$
\begin{gathered}
0<r_{i} \leq R_{i}, \quad r_{i} \leq\left(\eta_{i}^{\min }\right)^{1 /\left(1-\gamma_{i}\right)}, \\
R_{i} \geq \eta_{i}^{\max }|R|_{p}^{\gamma_{i}}+|r|_{p}^{-\beta_{i}} \max _{t \in[0, T]} \int_{0}^{T} g_{i}(t, s) w_{i}(s) d s,
\end{gathered}
$$

(here $|R|_{p}$ is the $l_{p}$ norm of $\left(R_{1}, R_{2}, \ldots, R_{n}\right)$, likewise $|r|_{p}$ is the $l_{p}$ norm of $\left.\left(r_{1}, r_{2}, \ldots, r_{n}\right)\right)$.

In proving Theorem 36, we actually seek a constant-sign solution of $(105)$ in $(C[0, T])^{n}$ and then extend it to a $T$ periodic constant-sign solution of (104) as in (106). Let $\Omega$ be the closed convex set given by

$$
\begin{aligned}
\Omega=\{u & \in(C[0, T])^{n} \mid r_{i} \leq \theta_{i} u_{i}(t) \leq R_{i}, t \in[0, T], \\
& 1 \leq i \leq n\},
\end{aligned}
$$

where $R_{i} \geq r_{i}>0$ are chosen as in (112) and (113), and define the operator $S: \Omega \rightarrow(C[0, T])^{n}$ as

$$
S u(t)=\left(S_{1} u(t), S_{2} u(t), \ldots, S_{n} u(t)\right), \quad t \in[0, T],
$$

where

$$
S_{i} u(t)=\int_{0}^{T} g_{i}(t, s) F_{i}(s, u(s)) d s, \quad t \in[0, T], 1 \leq i \leq n .
$$

Clearly, a fixed point of $S u=u$ is a solution of (105). The conditions of Theorem 26 are then shown to be satisfied.
Remark 37. As seen from (112) and (113), the functions $w_{i}$ and $q_{i}$ that appear in (C11) determine the lower and upper bounds of the solution $u_{i}^{T}, 1 \leq i \leq n$.

Theorem 38. Assume that the following conditions hold for each $1 \leq i \leq n$ : (C1), (C10), (C11), and (C12). Then, (104) has a T-periodic constant-sign solution $u^{T} \in(C(\mathbb{R}))^{n}$ such that

$$
r \leq \theta_{i} u_{i}^{T}(t) \leq R, \quad t \in \mathbb{R}, 1 \leq i \leq n,
$$

where $0<r \leq R$, and for all $1 \leq i \leq n$,

$$
\begin{gathered}
r \leq \begin{cases}\left(\eta_{i}^{\min } n^{\gamma_{i} / p}\right)^{1 /\left(1-\gamma_{i}\right)}, & 1 \leq p<\infty, \\
\left(\eta_{i}^{\min }\right)^{1 /\left(1-\gamma_{i}\right)}, & p=\infty,\end{cases} \\
R \geq\left\{\begin{array}{r}
R^{\gamma_{i}} \eta_{i}^{\max } n^{\gamma_{i} / p} \\
+r^{-\beta_{i}} n^{-\beta_{i} / p}\left[\max _{t \in[0, T]} \int_{0}^{T} g_{i}(t, s) w_{i}(s) d s\right], \\
R^{\gamma_{i}} \eta_{i}^{\max }+r^{-\beta_{i}}\left[\max _{t \in[0, T]} \int_{0}^{T} g_{i}(t, s) w_{i}(s) d s\right], \\
p=\infty .
\end{array}\right.
\end{gathered}
$$

Theorem 38 is obtained by similar arguments used in the derivation of Theorem 36, with a new $\Omega$ defined as

$$
\begin{gathered}
\Omega=\left\{u \in(C[0, T])^{n} \mid r \leq \theta_{i} u_{i}(t) \leq R, t \in[0, T],\right. \\
1 \leq i \leq n\},
\end{gathered}
$$

where $R \geq r>0$ are chosen as in (118) and (119).

Remark 39. Remark 37 also holds for Theorem 38. Further, comparing the bounds $r_{i}, R_{i}, 1 \leq i \leq n$ in Theorem 36 (see (112), (113)) with the bounds $r, R$ in Theorem 38 (see (118), (119)), we note that $r_{i}$ and $R_{i}$ are lower and upper bounds for a particular $\theta_{i} u_{i}^{T}$, whereas $r$ and $R$ are uniform lower and upper bounds for all $\theta_{i} u_{i}^{T}, 1 \leq i \leq n$. However, the computation of $R_{i}$ from (113) is more difficult than calculating $R$ from (119).

Our next result tackles the case when $\eta_{i}^{\min }=0$.

Theorem 40. Assume that the following conditions hold for each $1 \leq i \leq n$ : (C1), (C10),

(C13) there exist $\beta_{i} \in(0,1), \gamma_{i} \in[0,1)$, and T-periodic functions $w_{i}, v_{i}, q_{i}$ with $w_{i} \in L^{1}[0, T], v_{i} \in L^{1}[0, T], q_{i} \in$ $L^{1}[0, T]$, and $w_{i}(t), v_{i}(t)>0$ for a.e. $t \in[0, T]$ such that

$$
\begin{aligned}
\theta_{i} q_{i}(t)|u|^{\gamma_{i}}+v_{i}(t)|u|^{-\beta_{i}} \leq & \theta_{i} F_{i}(t, u) \\
\leq & \theta_{i} q_{i}(t)|u|^{\gamma_{i}}+w_{i}(t)|u|^{-\beta_{i}}, \\
& \quad t \in[0, T], u \in \prod_{k=1}^{n}(0, \infty)_{k}
\end{aligned}
$$

(here $|\cdot|$ is the $l_{p}$ norm where $1 \leq p \leq \infty$ is fixed); $(\mathrm{C} 14) \eta_{i}^{\min }=0$. 
Then, (104) has a T-periodic constant-sign solution $u^{T} \in$ $(C(\mathbb{R}))^{n}$ such that

$$
\frac{1}{R} \leq \theta_{i} u_{i}^{T}(t) \leq R, \quad t \in \mathbb{R}, 1 \leq i \leq n,
$$

where $R \geq 1$, and for all $1 \leq i \leq n$,

$$
\begin{gathered}
R \geq\left\{\begin{array}{c}
n^{\beta_{i} / p\left(1-\beta_{i}\right)}\left[\min _{t \in[0, T]} \int_{0}^{T} g_{i}(t, s) v_{i}(s) d s\right]^{-1 /\left(1-\beta_{i}\right)}, \\
{\left[\min _{t \in[0, T]} \int_{0}^{T} g_{i}(t, s) v_{i}(s) d s\right]_{p=\infty,}^{-1 /\left(1-\beta_{i}\right)},}
\end{array}\right. \\
R \geq\left\{\begin{array}{c}
R^{\gamma_{i}} \eta_{i}^{\max } n^{\gamma_{i} / p}+R^{\beta_{i}} n^{-\beta_{i} / p}\left[\max _{t \in[0, T]} \int_{0}^{T} g_{i}(t, s) w_{i}(s) d s\right], \\
R^{\gamma_{i}} \eta_{i}^{\max }+R^{\beta_{i}}\left[\max _{t \in[0, T]} \int_{0}^{T} g_{i}(t, s) w_{i}(s) d s\right], \\
p=\infty .
\end{array}\right.
\end{gathered}
$$

The closed convex set used to get Theorem 40 is given by

$$
\begin{gathered}
\Omega=\left\{u \in(C[0, T])^{n} \mid r \leq \theta_{i} u_{i}(t) \leq R,\right. \\
t \in[0, T], 1 \leq i \leq n\},
\end{gathered}
$$

where $r=1 / R$ and $R \geq 1$ satisfies (123).

Remark 41. As seen from (123), the functions $w_{i}, v_{i}$, and $q_{i}$ that appear in (C13) determine the lower and upper bounds of the solution $u_{i}^{T}, 1 \leq i \leq n$.

Finally, the next result tackles the case when $\eta_{i}^{\max }<0$.

Theorem 42. Assume that the following conditions hold for each $1 \leq i \leq n$ : (C1), (C10),

(C15) there exist $\beta \in(0,1)$ and $T$-periodic functions $w_{i}, v_{i}, q_{i}$ with $w_{i} \in L^{1}[0, T], v_{i} \in L^{1}[0, T], q_{i} \in L^{1}[0, T]$, and $w_{i}(t), v_{i}(t)>0$ for a.e. $t \in[0, T]$ such that

$$
\begin{array}{r}
\theta_{i} q_{i}(t)+v_{i}(t)|u|^{-\beta} \leq \theta_{i} F_{i}(t, u) \leq \theta_{i} q_{i}(t)+w_{i}(t)|u|^{-\beta}, \\
t \in[0, T], u \in \prod_{k=1}^{n}(0, \infty)_{k}
\end{array}
$$

(here $|\cdot|$ is the $l_{p}$ norm where $1 \leq p \leq \infty$ is fixed);

(C16) $\eta_{i}^{\max }<0$;

(C17) $\eta_{i}^{\min } \geq n^{-\beta /(1+\beta) p} W^{-\beta /\left(1-\beta^{2}\right)}\left(V \beta^{2}\right)^{1 /\left(1-\beta^{2}\right)}\left(1-1 / \beta^{2}\right)$ where

$$
\begin{gathered}
W=\max _{1 \leq k \leq n}\left[\max _{t \in[0, T]} \int_{0}^{T} g_{k}(t, s) w_{k}(s) d s\right], \\
V=\min _{1 \leq k \leq n}\left[\min _{t \in[0, T]} \int_{0}^{T} g_{k}(t, s) v_{k}(s) d s\right] .
\end{gathered}
$$

Then, (104) has a T-periodic constant-sign solution $u^{T} \in$ $(C(\mathbb{R}))^{n}$ such that

$$
r \leq \theta_{i} u_{i}^{T}(t) \leq R, \quad t \in \mathbb{R}, 1 \leq i \leq n,
$$

where $0<r \leq R$ are given by

$$
\begin{aligned}
& r= \begin{cases}n^{-\beta /(1+\beta) p} W^{-\beta /\left(1-\beta^{2}\right)}\left(V \beta^{2}\right)^{1 /\left(1-\beta^{2}\right)}, & 1 \leq p<\infty \\
W^{-\beta /\left(1-\beta^{2}\right)}\left(V \beta^{2}\right)^{1 /\left(1-\beta^{2}\right)}, & p=\infty,\end{cases} \\
& R= \begin{cases}n^{-\beta /(1+\beta) p} W^{1 /\left(1-\beta^{2}\right)}\left(V \beta^{2}\right)^{-\beta /\left(1-\beta^{2}\right)}, & 1 \leq p<\infty \\
W^{1 /\left(1-\beta^{2}\right)}\left(V \beta^{2}\right)^{-\beta /\left(1-\beta^{2}\right)}, & p=\infty .\end{cases}
\end{aligned}
$$

Theorem 42 is obtained by considering the closed convex set

$$
\begin{gathered}
\Omega=\left\{u \in(C[0, T])^{n} \mid r \leq \theta_{i} u_{i}(t) \leq R, t \in[0, T],\right. \\
1 \leq i \leq n\},
\end{gathered}
$$

where $R \geq r>0$ are determined later as those given in (128).

Remark 43. As seen from (128), the functions $w_{i}$ and $v_{i}$ that appear in (C15) determine the lower and upper bounds of the solution $u_{i}^{T}, 1 \leq i \leq n$.

We have so far established the results when (i) $\eta_{i}^{\min }>0$, (ii) $\eta_{i}^{\min }=0$, and (iii) $\eta_{i}^{\max }<0$ for all $1 \leq i \leq n$. However, it could be that we only have $\eta_{i}(t) \geq 0$ for some $i$ and $\eta_{j}(t)<0$ for some $j$, which results in $\eta_{i}^{\min } \geq 0$ and $\eta_{j}^{\max }<0$ for some $1 \leq i, j \leq n$. We present two results for such a case as follows. Note that Theorem 44 is obtained by applying Theorems 3842 , while Theorem 45 is obtained by applying Theorems 36 , 40 , and 42 .

Theorem 44. Let (C1) and (C10) hold for each $1 \leq i \leq n$. Assume the following:

(C18) conditions (C11) and (C12) hold for some $i \in I \subseteq$ $\{1,2, \ldots, n\}$

(C19) conditions (C13) and (C14) hold for some $i \in J \subseteq$ $\{1,2, \ldots, n\}$;

(C20) conditions (C15), (C16), and (C17) hold for some $i \epsilon$ $K \subseteq\{1,2, \ldots, n\}$

where $I \cup J \cup K=\{1,2, \ldots, n\}$. Then, (104) has a $T$-periodic constant-sign solution $u^{T} \in(C(\mathbb{R}))^{n}$ such that

$$
r \leq \theta_{i} u_{i}^{T}(t) \leq R, \quad t \in \mathbb{R}, 1 \leq i \leq n,
$$

where $0<r \leq R$ satisfy
(a) (118) and (119) for $i \in I$;
(b) $r=1 / R, R \geq 1$, (123) for $i \in J$;
(c) (128) for $i \in K$. 
Theorem 45. Let (C1) and (C10) hold for each $1 \leq i \leq n$. Assume that (C18)-(C20) hold with $I \cup J \cup K=\{1,2, \ldots, n\}$. Then, (104) has a T-periodic constant-sign solution $u^{T} \in$ $(C(\mathbb{R}))^{n}$ such that

$$
r_{i} \leq \theta_{i} u_{i}^{T}(t) \leq R_{i}, \quad t \in \mathbb{R}, i \in I,
$$

where $0<r_{i} \leq R_{i}$ satisfy (112) and (113) for $i \in I$, and

$$
r \leq \theta_{i} u_{i}^{T}(t) \leq R, \quad t \in \mathbb{R}, i \in J \cup K,
$$

where $0<r \leq R$ satisfy conclusions (b) and (c) of Theorem 44.

We will now apply the results obtained to the following system of Hill's equations, a particular form of it (see (64)) that has been discussed in [45],

$$
\begin{aligned}
& u_{1}^{\prime \prime}(t)+a_{1}(t) u_{1}(t)=\left(\sqrt{u_{1}^{2}+u_{2}^{2}}\right)^{-\beta_{1}}+v_{1}\left(\sqrt{u_{1}^{2}+u_{2}^{2}}\right)^{\gamma_{1}}, \\
& u_{2}^{\prime \prime}(t)+a_{2}(t) u_{2}(t)=\left(\sqrt{u_{1}^{2}+u_{2}^{2}}\right)^{-\beta_{2}}+v_{2}\left(\sqrt{u_{1}^{2}+u_{2}^{2}}\right)^{\gamma_{2}} .
\end{aligned}
$$

Clearly, the system (133) corresponds to (104) where $n=2$ and

$$
F_{i}(t, u)=\left(\sqrt{u_{1}^{2}+u_{2}^{2}}\right)^{-\beta_{i}}+v_{i}\left(\sqrt{u_{1}^{2}+u_{2}^{2}}\right)^{\gamma_{i}}, \quad i=1,2 .
$$

We will assume that $a_{1}, a_{2} \in L^{1}[0, T]$ satisfy (C1). Note that condition (C10) is clearly satisfied. Further, let $\theta_{1}=\theta_{2}=1$, that is, we are interested in positive periodic solutions of (133).

Example 46. Consider the system (133) with

$$
v_{i}>0, \quad \beta_{i}>0, \quad \gamma_{i} \in[0,1), \quad i=1,2 .
$$

Clearly, (C11) is satisfied with $p=2, q_{i}=v_{i}$ and $w_{i}=$ $1, i=1,2$. Thus, (C12) also holds since

$$
\begin{aligned}
\eta_{i}^{\min } & =\min _{t \in[0, T]} \int_{0}^{T} g_{i}(t, s) \theta_{i} q_{i}(s) d s \\
& =v_{i} \min _{t \in[0, T]} \int_{0}^{T} g_{i}(t, s) d s>0 .
\end{aligned}
$$

Theorem 38 (or Theorem 36) is applicable and we conclude that the system (133) with (135) has a $T$-periodic positive solution $u^{T} \in(C(\mathbb{R}))^{2}$ such that

$$
r \leq u_{i}^{T}(t) \leq R, \quad t \in \mathbb{R}, i=1,2,
$$

where $0<r \leq R$ are such that

$$
\begin{gathered}
r \leq \min _{i=1,2}\left\{\left(\eta_{i}^{\min } 2^{\gamma_{i} / 2}\right)^{1 /\left(1-\gamma_{i}\right)}\right\} \\
R \geq \max _{i=1,2}\left\{R^{\gamma_{i}} \eta_{i}^{\max } 2^{\gamma_{i} / 2}+r^{-\beta_{i}} 2^{-\beta_{i} / 2}\left[\max _{t \in[0, T]} \int_{0}^{T} g_{i}(t, s) d s\right]\right\} .
\end{gathered}
$$

To illustrate numerically, suppose

$$
\begin{gathered}
a_{1}(t)=a_{2}(t)=\frac{1}{4}, \quad T=2 \pi, \quad \nu_{1}=\frac{1}{4}, \\
v_{2}=1, \quad \gamma_{1}=\gamma_{2}=\frac{1}{2}, \quad \beta_{1}=\beta_{2}=1 .
\end{gathered}
$$

Green's function is given in (95) and

$$
\eta_{i}^{\min }=v_{i} \min _{t \in[0, T]} \int_{0}^{T} g_{i}(t, s) d s=4 v_{i}
$$

Hence, (138) yields $r \leq \sqrt{2}$. Let $r=\sqrt{2}$, then (139) reduces to

$$
R \geq \max _{i=1,2}\left\{R^{1 / 2} 4 v_{i} 2^{1 / 4}+r^{-1} 2^{-1 / 2} 4 v_{i}\right\}=R^{1 / 2} 2^{9 / 4}+2,
$$

which is satisfied by $R \geq 26.48$. Let $R=26.48$, then from (137) we conclude that the system (133) with (140) has a $2 \pi$-periodic positive solution $u \in(C(\mathbb{R}))^{2}$ such that

$$
\sqrt{2} \leq u_{i}(t) \leq 26.48, \quad t \in \mathbb{R}, i=1,2 .
$$

Example 47. Consider the system (133) with

$$
v_{i}=0, \quad \beta_{i} \in(0,1), \quad \gamma_{i} \in[0,1), \quad i=1,2 .
$$

Here, (C13) is satisfied with $p=2, q_{i}=v_{i}=0$ and $w_{i}=$ $v_{i}=1, i=1,2$. Subsequently, (C14) also holds since

$$
\begin{aligned}
\eta_{i}^{\min } & =\min _{t \in[0, T]} \int_{0}^{T} g_{i}(t, s) \theta_{i} q_{i}(s) d s \\
& =v_{i} \min _{t \in[0, T]} \int_{0}^{T} g_{i}(t, s) d s=0 .
\end{aligned}
$$

Employing Theorem 40, we conclude that the system (133) with (144) has a $T$-periodic positive solution $u^{T} \in(C(\mathbb{R}))^{2}$ such that

$$
\frac{1}{R} \leq u_{i}^{T}(t) \leq R, \quad t \in \mathbb{R}, i=1,2,
$$

where $R \geq 1$, and from (123), we have for $i=1,2$,

$$
\begin{aligned}
& R \geq 2^{\beta_{i} / 2\left(1-\beta_{i}\right)}\left[\min _{t \in[0, T]} \int_{0}^{T} g_{i}(t, s) d s\right]^{-1 /\left(1-\beta_{i}\right)}, \\
& R \geq\left\{2^{-\beta_{i} / 2}\left[\max _{t \in[0, T]} \int_{0}^{T} g_{i}(t, s) d s\right]\right\}^{1 /\left(1-\beta_{i}\right)} .
\end{aligned}
$$

Combining the inequalities, we see that $R$ should satisfy

$$
\begin{aligned}
R \geq \max \left\{1, \max _{i=1,2} 2^{\beta_{i} / 2\left(1-\beta_{i}\right)}\right. & \\
& \times\left[\min _{t \in[0, T]} \int_{0}^{T} g_{i}(t, s) d s\right]^{-1 /\left(1-\beta_{i}\right)}, \\
& \left.\max _{i=1,2}\left\{2^{-\beta_{i} / 2}\left[\max _{t \in[0, T]} \int_{0}^{T} g_{i}(t, s) d s\right]\right\}^{1 /\left(1-\beta_{i}\right)}\right\} .
\end{aligned}
$$


Example 48. Consider the system (133) with

$$
\begin{aligned}
& v_{i}<0, \quad \beta_{i}=\beta \in(0,1), \quad \gamma_{i}=0, \quad i=1,2 \\
& v_{i} \min _{t \in[0, T]} \int_{0}^{T} g_{i}(t, s) d s \geq 2^{-\beta / 2(1+\beta)} W^{-\beta /\left(1-\beta^{2}\right)}\left(V \beta^{2}\right)^{1 /\left(1-\beta^{2}\right)} \\
& \times\left(1-\frac{1}{\beta^{2}}\right), \quad i=1,2
\end{aligned}
$$

where

$$
\begin{aligned}
& W=\max _{k=1,2}\left[\max _{t \in[0, T]} \int_{0}^{T} g_{k}(t, s) d s\right], \\
& V=\min _{k=1,2}\left[\min _{t \in[0, T]} \int_{0}^{T} g_{k}(t, s) d s\right] .
\end{aligned}
$$

Obviously, (C15) is satisfied with $p=2, q_{i}=v_{i}<0$ and $w_{i}=v_{i}=1, i=1,2$. Then, (C16) also holds since

$$
\begin{aligned}
\eta_{i}^{\max } & =\max _{t \in[0, T]} \int_{0}^{T} g_{i}(t, s) \theta_{i} q_{i}(s) d s \\
& =v_{i} \max _{t \in[0, T]} \int_{0}^{T} g_{i}(t, s) d s<0 .
\end{aligned}
$$

Moreover, condition (C17) is simply (150). Hence, we conclude from Theorem 42 that the system (133) with (149) and (150) has a $T$-periodic positive solution $u^{T} \in(C(\mathbb{R}))^{2}$ such that

$$
r \leq u_{i}^{T}(t) \leq R, \quad t \in \mathbb{R}, i=1,2,
$$

where $0<r \leq R$ are given by

$$
\begin{aligned}
& r=2^{-\beta / 2(1+\beta)} W^{-\beta /\left(1-\beta^{2}\right)}\left(V \beta^{2}\right)^{1 /\left(1-\beta^{2}\right)}, \\
& R=2^{-\beta / 2(1+\beta)} W^{1 /\left(1-\beta^{2}\right)}\left(V \beta^{2}\right)^{-\beta /\left(1-\beta^{2}\right)} .
\end{aligned}
$$

Remark 49. In [45], it is shown that (64) has a solution when $\beta>0, \gamma \in[0,1)$ and $v>0$. As seen from Examples 46-48, we have generalized the system (64) to allow $v$ to be zero or negative.

\section{Acknowledgments}

J. Chu was supported by the National Natural Science Foundation of China (Grant nos. 11171090 and 11271078), the Program for New Century Excellent Talents in University (Grant no. NCET-10-0325), and China Postdoctoral Science Foundation funded project (Grant no. 2012T50431). J. Sun was supported by the National Natural Science Foundation of China (Grant nos. 11201270 and 11271372), Shandong Natural Science Foundation (Grant no. ZR2012AQ010), and Young Teacher Support Program of Shandong University of Technology.

\section{References}

[1] R. P. Agarwal, D. Franco, and D. O'Regan, "Singular boundary value problems for first and second order impulsive differential equations," Aequationes Mathematicae, vol. 69, no. 1-2, pp. 8396, 2005.

[2] R. P. Agarwal, D. O’Regan, and B. Yan, "Multiple positive solutions of singular Dirichlet second-order boundary-value problems with derivative dependence," Journal of Dynamical and Control Systems, vol. 15, no. 1, pp. 1-26, 2009.

[3] A. Ambrosetti and V. Coti Zelati, Periodic Solutions of Singular Lagrangian Systems, Birkhäuser Boston Inc., Boston, Mass, USA, 1993.

[4] A. Bahri and P. H. Rabinowitz, "A minimax method for a class of Hamiltonian systems with singular potentials," Journal of Functional Analysis, vol. 82, no. 2, pp. 412-428, 1989.

[5] I. V. Barteneva, A. Cabada, and A. O. Ignatyev, "Maximum and anti-maximum principles for the general operator of second order with variable coefficients," Applied Mathematics and Computation, vol. 134, no. 1, pp. 173-184, 2003.

[6] A. Boucherif and N. Daoudi-Merzagui, "Periodic solutions of singular nonautonomous second order differential equations," NoDEA. Nonlinear Differential Equations and Applications, vol. 15, no. 1-2, pp. 147-158, 2008.

[7] J. Chu and J. J. Nieto, "Recent existence results for secondorder singular periodic differential equations," Boundary Value Problems, vol. 2009, Article ID 540863, 20 pages, 2009.

[8] P. Habets and L. Sanchez, "Periodic solutions of some Liénard equations with singularities," Proceedings of the American Mathematical Society, vol. 109, no. 4, pp. 1035-1044, 1990.

[9] X. Li and Z. Zhang, "Periodic solutions for damped differential equations with a weak repulsive singularity," Nonlinear Analysis. Theory, Methods \& Applications, vol. 70, no. 6, pp. 2395-2399, 2009.

[10] I. Rachunkova and M. Tvrdý, "Existence results for impulsive second-order periodic problems," Nonlinear Analysis. Theory, Methods \& Applications, vol. 59, no. 1-2, pp. 133-146, 2004.

[11] P. Yan and M. Zhang, "Higher order non-resonance for differential equations with singularities," Mathematical Methods in the Applied Sciences, vol. 26, no. 12, pp. 1067-1074, 2003.

[12] V. Bevc, J. L. Palmer, and C. Susskind, "On the design of the transition region of axisymmetric, magnetically focused beam valves," Journal of the British Institution of Radio Engineers, vol. 18, no. 12, pp. 696-708, 1958.

[13] J. Ren, Z. Cheng, and S. Siegmund, "Positive periodic solution for Brillouin electron beam focusing system," Discrete and Continuous Dynamical Systems B, vol. 16, no. 1, pp. 385-392, 2011.

[14] P. J. Torres, "Existence and uniqueness of elliptic periodic solutions of the Brillouin electron beam focusing system," Mathematical Methods in the Applied Sciences, vol. 23, no. 13, pp. 1139-1143, 2000.

[15] M. A. del Pino and R. F. Manásevich, "Infinitely many Tperiodic solutions for a problem arising in nonlinear elasticity," Journal of Differential Equations, vol. 103, no. 2, pp. 260-277, 1993.

[16] J. Chu and M. Zhang, "Rotation numbers and Lyapunov stability of elliptic periodic solutions," Discrete and Continuous Dynamical Systems A, vol. 21, no. 4, pp. 1071-1094, 2008.

[17] J. Chu and M. Li, "Twist periodic solutions of second order singular differential equations," Journal of Mathematical Analysis and Applications, vol. 355, no. 2, pp. 830-838, 2009. 
[18] M. Zhang, "Periodic solutions of equations of Emarkov-Pinney type," Advanced Nonlinear Studies, vol. 6, no. 1, pp. 57-67, 2006.

[19] J. Sun and D. O’Regan, "Impulsive periodic solutions for singular problems via variational methods," Bulletin of the Australian Mathematical Society, vol. 86, no. 2, pp. 193-204, 2012.

[20] J. Sun, J. Chu, and H. Chen, "Periodic solution generated by impulses for singular differential equations," In press.

[21] D. Bonheure and C. De Coster, "Forced singular oscillators and the method of lower and upper solutions," Topological Methods in Nonlinear Analysis, vol. 22, no. 2, pp. 297-317, 2003.

[22] J. L. Bravo and P. J. Torres, "Periodic solutions of a singular equation with indefinite weight," Advanced Nonlinear Studies, vol. 10, no. 4, pp. 927-938, 2010.

[23] J. Chu and J. J. Nieto, "Impulsive periodic solutions of firstorder singular differential equations," Bulletin of the London Mathematical Society, vol. 40, no. 1, pp. 143-150, 2008.

[24] A. Fonda and R. Toader, "Periodic orbits of radially symmetric Keplerian-like systems: a topological degree approach," Journal of Differential Equations, vol. 244, no. 12, pp. 3235-3264, 2008.

[25] A. Fonda and A. J. Ureña, "Periodic, subharmonic, and quasiperiodic oscillations under the action of a central force," Discrete and Continuous Dynamical Systems A, vol. 29, no. 1, pp. 169-192, 2011.

[26] P. J. Torres, "Non-collision periodic solutions of forced dynamical systems with weak singularities," Discrete and Continuous Dynamical Systems. Series A, vol. 11, no. 2-3, pp. 693-698, 2004.

[27] M. Zhang, "Periodic solutions of damped differential systems with repulsive singular forces," Proceedings of the American Mathematical Society, vol. 127, no. 2, pp. 401-407, 1999.

[28] M. Zhang, "A relationship between the periodic and the Dirichlet BVPs of singular differential equations," Proceedings of the Royal Society of Edinburgh A, vol. 128, no. 5, pp. 1099-1114, 1998.

[29] A. C. Lazer and S. Solimini, "On periodic solutions of nonlinear differential equations with singularities," Proceedings of the American Mathematical Society, vol. 99, no. 1, pp. 109-114, 1987.

[30] W. B. Gordon, "Conservative dynamical systems involving strong forces," Transactions of the American Mathematical Society, vol. 204, pp. 113-135, 1975.

[31] J. Chu and P. J. Torres, "Applications of Schauder's fixed point theorem to singular differential equations," Bulletin of the London Mathematical Society, vol. 39, no. 4, pp. 653-660, 2007.

[32] J. Chu, P. J. Torres, and M. Zhang, "Periodic solutions of second order non-autonomous singular dynamical systems," Journal of Differential Equations, vol. 239, no. 1, pp. 196-212, 2007.

[33] J. Chu and M. Li, "Positive periodic solutions of Hill's equations with singular nonlinear perturbations," Nonlinear Analysis. Theory, Methods \& Applications, vol. 69, no. 1, pp. 276-286, 2008.

[34] J. Chu and Z. Zhang, "Periodic solutions of second order superlinear singular dynamical systems," Acta Applicandae Mathematicae, vol. 111, no. 2, pp. 179-187, 2010.

[35] J. Chu, N. Fan, and P. J. Torres, "Periodic solutions for second order singular damped differential equations," Journal of Mathematical Analysis and Applications, vol. 388, no. 2, pp. 665-675, 2012.

[36] D. Franco and P. J. Torres, "Periodic solutions of singular systems without the strong force condition," Proceedings of the American Mathematical Society, vol. 136, no. 4, pp. 1229-1236, 2008.

[37] D. Jiang, J. Chu, and M. Zhang, "Multiplicity of positive periodic solutions to superlinear repulsive singular equations," Journal of Differential Equations, vol. 211, no. 2, pp. 282-302, 2005.
[38] I. Rachunkova, M. Tvrdý, and I. Vrkoč, "Existence of nonnegative and nonpositive solutions for second order periodic boundary value problems," Journal of Differential Equations, vol. 176, no. 2, pp. 445-469, 2001.

[39] P. J. Torres, "Weak singularities may help periodic solutions to exist," Journal of Differential Equations, vol. 232, no. 1, pp. 277284, 2007.

[40] A. Cabada and J. Cid, "On the sign of the Green's function associated to Hill's equation with an indefinite potential," Applied Mathematics and Computation, vol. 205, no. 1, pp. 303308, 2008.

[41] Z. Cheng and J. Ren, "Periodic and subharmonic solutions for Duffing equation with a singularity," Discrete and Continuous Dynamical Systems. Series A, vol. 32, no. 5, pp. 1557-1574, 2012.

[42] J. Chu and Z. Zhang, "Periodic solutions of singular differential equations with sign-changing potential," Bulletin of the Australian Mathematical Society, vol. 82, no. 3, pp. 437-445, 2010.

[43] D. Franco and J. R. L. Webb, "Collisionless orbits of singular and non singular dynamical systems," Discrete and Continuous Dynamical Systems A, vol. 15, no. 3, pp. 747-757, 2006.

[44] R. Hakl and P. J. Torres, "On periodic solutions of second-order differential equations with attractive-repulsive singularities," Journal of Differential Equations, vol. 248, no. 1, pp. 111-126, 2010.

[45] X. Lin, D. Jiang, D. O'Regan, and R. P. Agarwal, "Twin positive periodic solutions of second order singular differential systems," Topological Methods in Nonlinear Analysis, vol. 25, no. 2, pp. 263-273, 2005.

[46] P. J. Torres, "Existence of one-signed periodic solutions of some second-order differential equations via a Krasnoselskii fixed point theorem," Journal of Differential Equations, vol. 190, no. 2, pp. 643-662, 2003.

[47] M. Zhang and W. Li, "A Lyapunov-type stability criterion using $L^{\infty}$ norms," Proceedings of the American Mathematical Society, vol. 130, no. 11, pp. 3325-3333, 2002.

[48] M. Zhang, "Optimal conditions for maximum and antimaximum principles of the periodic solution problem," Boundary Value Problems, vol. 2010, Article ID 410986, 26 pages, 2010.

[49] A. Granas, R. B. Guenther, and J. W. Lee, "Some general existence principles in the Carathéodory theory of nonlinear differential systems," Journal de Mathématiques Pures et Appliquées, vol. 70, no. 2, pp. 153-196, 1991.

[50] A. Granas and J. Dugundji, Fixed Point Theory, Springer Monographs in Mathematics, Springer-Verlag, New York, NY, USA, 2003.

[51] M. A. Krasnosel'skii, Positive Solutions of Operator Equations, P. Noordhoff Ltd., Groningen, TheNetherlands, 1964.

[52] R. Hakl and P. J. Torres, "Maximum and antimaximum principles for a second order differential operator with variable coefficients of indefinite sign," Applied Mathematics and Computation, vol. 217, no. 19, pp. 7599-7611, 2011.

[53] J. J. Nieto and D. O’Regan, "Variational approach to impulsive differential equations," Nonlinear Analysis. Real World Applications, vol. 10, no. 2, pp. 680-690, 2009.

[54] J. Sun, H. Chen, J. J. Nieto, and M. Otero-Novoa, "The multiplicity of solutions for perturbed second-order Hamiltonian systems with impulsive effects," Nonlinear Analysis. Theory, Methods \& Applications, vol. 72, no. 12, pp. 4575-4586, 2010.

[55] J. Mawhin and M. Willem, Critical Point Theory and Hamiltonian Systems, vol. 74, Springer-Verlag, New York, NY, USA, 1989.

[56] R. P. Agarwal, D. O’Regan, and P. J. Y. Wong, “Constant-sign solutions of a system of integral equations: the semipositone and 
singular case," Asymptotic Analysis, vol. 43, no. 1-2, pp. 47-74, 2005.

[57] R. P. Agarwal, D. O’Regan, and P. J. Y. Wong, "Constantsign solutions of a system of integral equations with integrable singularities," Journal of Integral Equations and Applications, vol. 19, no. 2, pp. 117-142, 2007.

[58] R. P. Agarwal, D. O'Regan, and P. J. Y. Wong, "Constant sign solutions for systems of Fredholm and Volterra integral equations: the singular case," Acta Applicandae Mathematicae, vol. 103, no. 3, pp. 253-276, 2008.

[59] R. P. Agarwal, D. O’Regan, and P. J. Y. Wong, "Constantsign solutions for systems of singular integral equations of Hammerstein type," Mathematical and Computer Modelling, vol. 50, no. 7-8, pp. 999-1025, 2009.

[60] R. P. Agarwal, D. O’Regan, and P. J. Y. Wong, “Constant-sign solutions for singular systems of Fredholm integral equations," Mathematical Methods in the Applied Sciences, vol. 33, no. 15, pp. 1783-1793, 2010.

[61] R. P. Agarwal, D. O’Regan, and P. J. Y. Wong, "Periodic constantsign solutions for systems of Hill's equations," Asymptotic Analysis, vol. 67, no. 3-4, pp. 191-216, 2010.

[62] D. O'Regan and M. Meehan, Existence Theory for Nonlinear Integral and Integrodifferential Equations, Kluwer, Dordrecht, The Netherlands, 1998. 


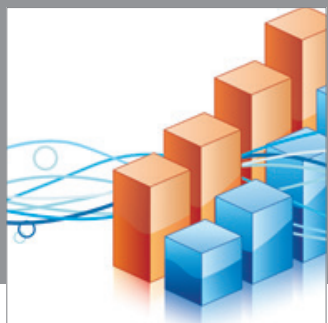

Advances in

Operations Research

mansans

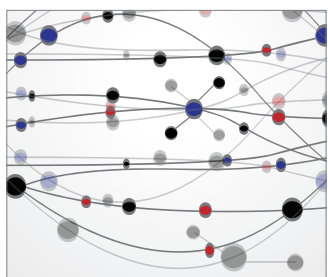

The Scientific World Journal
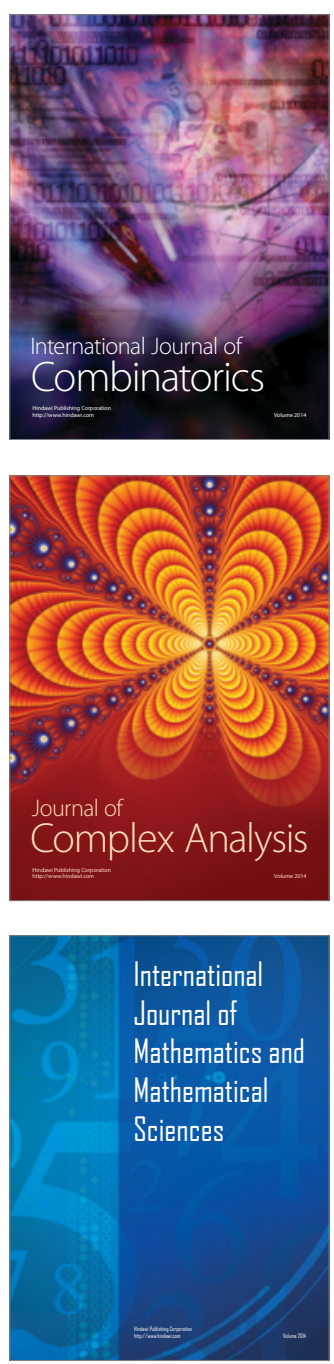
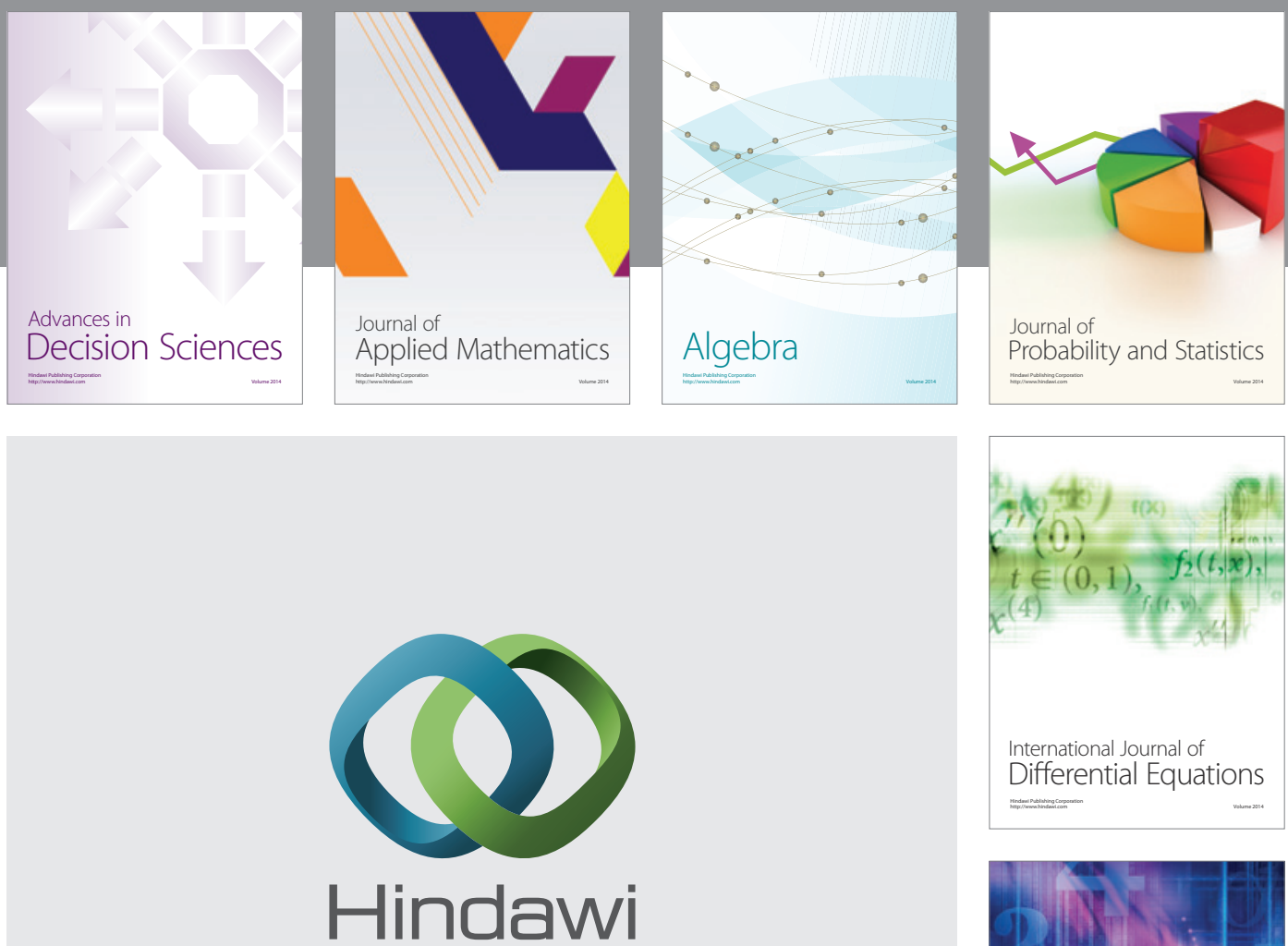

Submit your manuscripts at http://www.hindawi.com
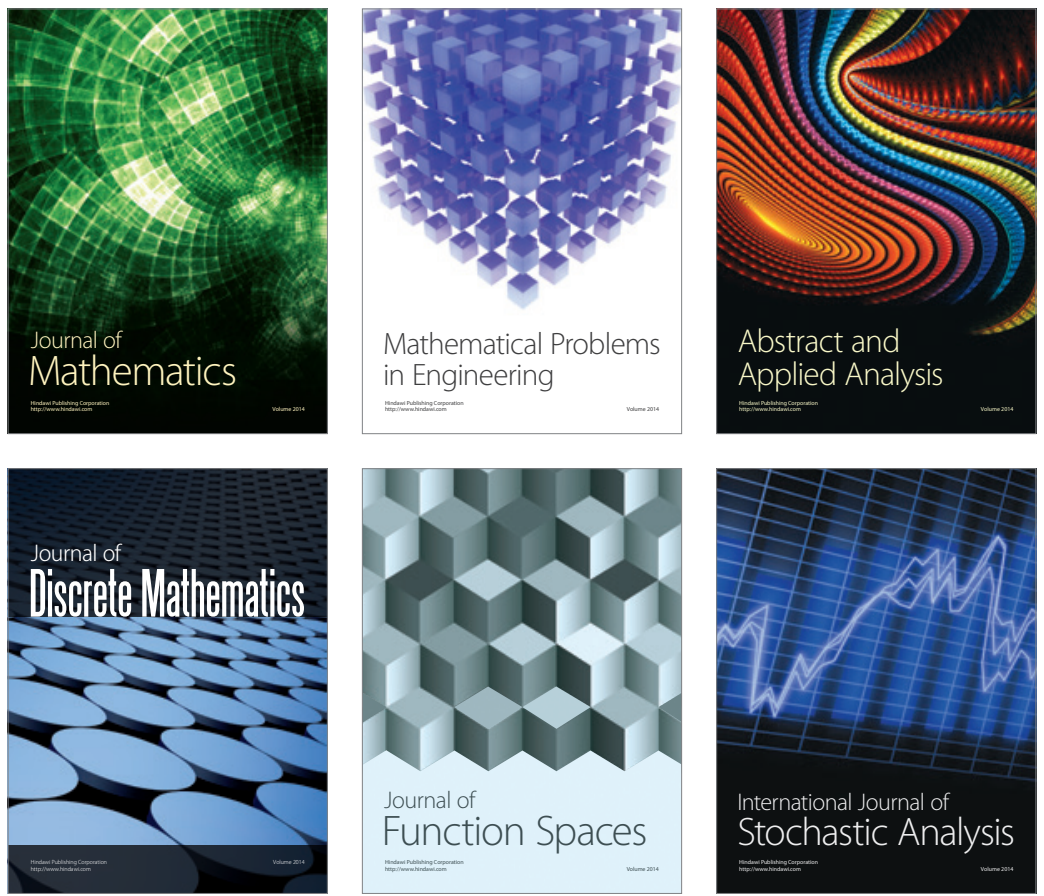

Journal of

Function Spaces

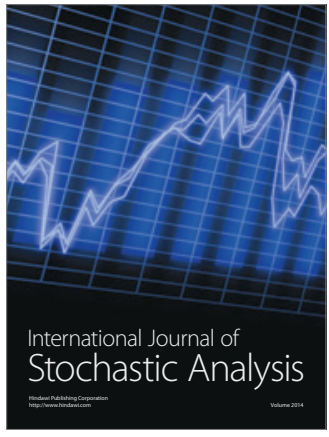

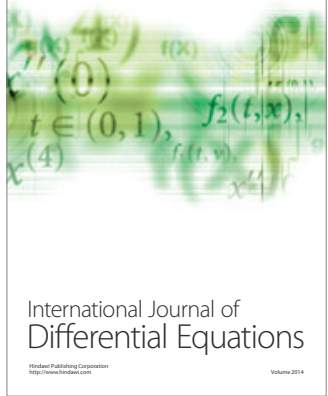
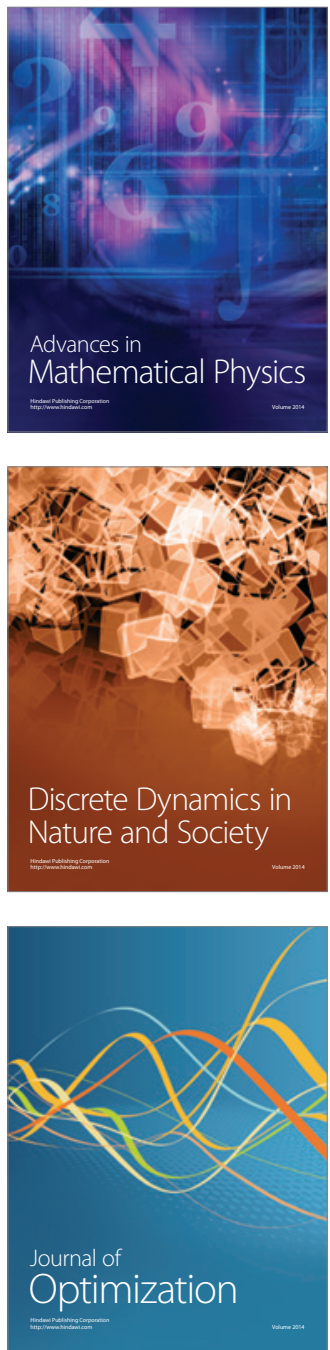